\title{
Inhibit inflammation and apoptosis of pyrroloquinoline on spinal cord injury in rat
}

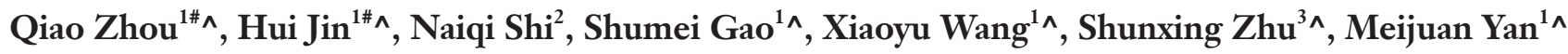 \\ ${ }^{1}$ The Key Laboratory of Neuroregeneration of Jiangsu and Ministry of Education, Co-innovation Center of Neuroregeneration, Nantong University, \\ Nantong, China; ${ }^{2}$ School of Chemistry and Molecular Biosciences, the University of Queensland, Brisbane, Australia; ${ }^{3}$ Experimental Animal Center \\ of Nantong University, Nantong, China \\ Contributions: (I) Conception and design: S Gao, Q Zhou, S Zhu, M Yan; (II) Administrative support: None; (III) Provision of study materials or \\ patients: H Jin, N Shi, X Wang; (IV) Collection and assembly of data: H Jin, N Shi, X Wang; (V) Data analysis and interpretation: S Gao, Q Zhou, S \\ Zhu, M Yan; (VI) Manuscript writing: All authors; (VII) Final approval of manuscript: All authors. \\ \#These authors contributed equally to this work. \\ Correspondence to: Meijuan Yan. The Key Laboratory of Neuroregeneration of Jiangsu and Ministry of Education, Co-innovation Center of \\ Neuroregeneration, Nantong University, 19 Qi-xiu Road, Nantong 226001, China. Email: ymz@ntu.edu.cn; Shunxing Zhu. Experimental Animal \\ Center of Nantong University, 19 Qi-xiu Road, Nantong 226001, China. Email: zsx@ntu.edu.cn.
}

Background: Pyrroloquinoline quinone (PQQ) is a redox cofactor that can participate in a variety of physiological and biochemical processes, such as anti-inflammatory, cytoprotection, anti-aging, and antiapoptosis. PQQ plays an important protective role in the central nervous system (CNS). However, the effects of PQQ on astrocytes of the CNS and spinal cord injury (SCI) of rats is still unclear. The present study investigates the role of PQQ in inflammation, apoptosis, and autophagy after SCI in rats. And the effect of PQQ on lipopolysaccharide (LPS)-induced apoptosis and inflammation of astrocytes in vitro, to explore the neuroprotective mechanism of PQQ.

Methods: Sixty specific pathogen free (SPF) SD male rats (200-250 g) were randomly divided into Normal group, Sham group, SCI group, and SCI + PQQ group, with 15 rats in each group. BBB score, HE staining, Nissl staining, Western blot, immunofluorescence, and other methods were used for detection.

Results: Our results showed that PQQ could upregulate BBB score in SCI rats. In the second place, PQQ can increase the number and improve the morphology of neurons after SCI. The expression of IL-1 $\beta$, TNF- $\alpha$, IL-6 was significantly decreased after PQQ treatment. And then, the ratio of B-cell lymphoma-2 (Bcl-2)/Bcl-2 associated $\mathrm{X}$ protein (Bax) increased significantly, and the positive signal of NeuN increased obviously after PQQ treatment. There are a large number of co-localizations between Bcl-2 and NeuN. Meanwhile, PQQ could down-regulate the expression of Active-Caspase3, and PQQ treatment could reverse the transfer of Active-Caspase3/Caspase3 from the cytoplasm to the nucleus in neurons and astrocytes after SCI. At the same time, PQQ had no significant effect on the LC3b/a ratio. PQQ could decrease the LAMP2 expression in spinal cord after injury. The expression level of phospho-Akt (p-AKT) increased after SCI and decreased after PQQ treatment. In primary astrocytes, LPS could induce the expression levels of IL-1 $\beta$, TNF- $\alpha$, and IL-6, and which were inhibited by PQQ treatment at 12 hours. After treatment with LPS, the expression level of Active-Caspase 3 increased, which could be reversed by PQQ treatment for $24 \mathrm{~h}$.

Conclusions: These results suggest that PQQ can ameliorate the motor function of hind limbs and the pathological changes of neurons and injured spinal cord after SCI, down-regulate the expressions of IL$1 \beta$, TNF- $\alpha$, and IL-6, inhibit apoptosis after SCI, and inhibit LPS-induced apoptosis and inflammation of astrocytes.

\footnotetext{
^ ORCID: Qiao Zhou, 0000-0002-0445-4742; Hui Jin, 0000-0002-3204-0782; Shumei Gao, 0000-0003-2751-1882; Xiaoyu Wang, 00000001-9113-5960; Shunxing Zhu, 0000-0001-8011-4099; Meijuan Yan, 0000-0002-6087-969X.
} 
Keywords: Pyrroloquinoline quinone (PQQ); spinal cord injury (SCI); apoptosis; autophagy; astrocytes

Submitted Apr 18, 2021. Accepted for publication Jul 16, 2021.

doi: $10.21037 /$ atm-21-1951

View this article at: https://dx.doi.org/10.21037/atm-21-1951

\section{Introduction}

Pyrroloquinoline quinone (PQQ) is an important nutrient existing in foods such as vegetables and meat (1-4), which cannot be synthesized by biology, but trace PQQ is detected in humans and rats (5-7). PQQ is a redox cofactor that can participate in a variety of physiological and biochemical processes in bacteria and mammals, such as preventing liver injury (8), preventing myocardial ischemiareperfusion injury, stimulating the production of nerve growth factor (NGF) in astrocytes, and eliminating free radicals (9). Studies have shown that the loss of PQQ in mammals will lead to systemic reactions such as weakened immune response, growth disorder, reduced respiratory entropy, and abnormal reproductive function (10-12). PQQ is a multifunctional biological agent with the effects of antioxidant growth, anti-inflammatory, anti-cancer, and aging $(2,13-16)$. The role of PQQ in cultured cells and animal models involves the activation and expression of cyclic adenosine monophosphate (cAMP) response element, mitochondria, peroxisome proliferator-activated receptor, and other nutritional factors $(17,18)$; these co-activators and transcription factors can regulate the biological origin of mitochondria and cell energy metabolism (17-20). PQQ can enhance the production of NGF and play a role in nerve protection $(15,21,22)$.

Spinal cord injury (SCI) is a disease that causes huge economic losses to patients and the health care system (23). Ninety percent of SCI patients are traumatic injuries caused by violence, exercise, traffic accidents, or falls, which usually results in loss of all sensory and/or motor functions or even paralysis (24). In SCI, the initial mechanical force is called primary injury (25-27). The secondary injury starts within minutes after the primary injury by mechanical force and will continue for weeks or months, making the injury spread from the lesion site to the surrounding crushed tissue (25). The secondary injury can occur inflammation, edema, neurotransmitter accumulation (excitotoxicity), necrotic cell death, and vascular injury $(25,28)$; Axon death, glial scar evolution around the injury site, demyelination of surviving axons and apoptosis occur (23). Mouse SCI models have been widely used. Metz and his colleagues compared the function and anatomical structure of human chronic SCI and rats contusion injury (29), using high-resolution magnetic resonance imaging (MRI) evaluation in human and rat contusion injury, found that the neuroanatomical changes and electrophysiological function are related (29), which proves that the rats SCI model can be used as an experimental model to study the biological function of SCI. Basso Beattie Bresnahan Scoring Assay (BBB) scale is a scale related to histopathological changes after SCI. It distinguishes the recovery stage of SCI according to the injury time or the recovery degree injury, which is 21 subscales (30-32). Therefore, the BBB score was used to evaluate the recovery of rats after SCI in this study.

Apoptosis is a kind of energy-dependent programmed death, which occurs within a few hours after primary SCI (25). In the rats SCI model, apoptosis begins at $4 \mathrm{~h}$ after injury and reaches the peak at $7 \mathrm{~d}$ (33). The main pathological feature of SCI is neuronal apoptosis (34). The induction pathways of apoptosis can be divided into exogenous and endogenous pathways in terms of triggering mechanisms (35). The exogenous pathway is activated by the combination of factors associated with apoptosis (FAS), tumor necrosis factor receptor 1 (TNFR1), and other death receptors with specific ligands, Caspase 3 is lysed to form phosphorylated Active-Caspase3, leading to the degradation of cells in the final stage of apoptosis (36). The endogenous pathway is induced by the balance between intracellular anti-apoptotic proteins, and pro-apoptotic proteins, and triggered by activation of Caspase 9 and mitochondrial release of cytochrome $\mathrm{C}$ (37). Bcl-2 superfamily members play an important role in maintaining the integrity of mitochondria, which are mainly divided into two categories: Bak and Bax that promote apoptosis (38), and Bcl-2 and $\mathrm{Bcl}-\mathrm{xL}$ that resist apoptosis. The endogenous pathway is triggered by the dimerization of pro-apoptotic Bak and Bax into the mitochondrial outer membrane. The apoptotic body activates Caspase 9 and lyses Caspase 3 to form ActiveCaspase3 for promoting the execution of apoptosis (39). Caspase 3 is the intersection of exogenous and endogenous pathways that induce apoptosis, indicating that the study of Caspase3 changes in apoptosis after SCI is expected to 
be used for the treatment of SCI (23). Therefore, in the present study, apoptosis-related proteins such asCaspase3, Active-Caspase3, Bcl-2, and Bax were used to clarify the effect of PQQ on apoptosis after SCI.

Autophagy plays an important role in intracellular homeostasis by assisting the conversion of organelles and proteins. Lysosomes in cells can degrade excess or harmful organelles and cytoplasmic proteins $(40,41)$. Autophagy has neuroprotective effects $(39,42)$, which can promote cell survival by removing damaged mitochondria and harmful proteins in the body (43). For a long time, microtubule-associated protein 1 light chain 3 (LC3) has been considered as a specific marker of autophagy. It can bind to the lipid phosphatidylethanolamine on the growing isolation membrane through the ubiquitin-binding system, and then interacts with the protein containing the LC3 interaction domain to form an LC3 positive isolation membrane and seal the phage to form a double-membrane autophagosome (44-46). Lysosome-associated membrane protein 2 (LAMP2) has the stability of lysosomes and protects the lysosome membrane from hydrolysis $(47,48)$. LAMP2 can mediate oxidative stress cell death in the central nervous system (CNS). It has been reported that the concentration of LAMP2 in cerebrospinal fluid of patients with Parkinson's disease (PD) decreased (49), and increased in patients with Alzheimer's disease (AD) (50,51). LC3mediated double-membrane autophagosomes can bind to lysosomes and produce autolysosomes, indicating that LC3 and LAMP2 play a very important role in the CNS system. Interestingly, autophagy has two-way functions. Studies have shown that autophagy under physiological conditions is conducive to the cell protection of ischemic SCI (52), while other studies have shown that excessive autophagy can lead to cell death (53). Although the mechanism of autophagy after SCI is not clear, autophagy can eliminate excess and harmful proteins and cells in the body to maintain environmental homeostasis. Autophagy has been considered a beneficial mechanism after SCI (23).

Astrocytes are a kind of nerve cells derived from the ectoderm and neuroepithelium, which can maintain the CNS defense function and homeostasis of the internal environment (54). They provide growth and nutritional factors for neurons and remove glutamate, ions, and other neurotransmitters in the extracellular environment (55). They play an important role in the CNS, including the reconstruction of the blood-brain barrier and the transport and maintenance of immune cell activity (56). Astrocytes can activate different intracellular signaling pathways after
SCI and participate in immune response (56). After SCI, activated glial cells produce several inflammatory factors, such as interleukin-1 $\beta$ (IL-1 $\beta$ ), interleukin-6 (IL-6), and tumor necrosis factor (TNF- $\alpha$ ). IL- $1 \beta$ can induce an inflammatory response and has a variety of characteristics for injury, infection, and immune response (57). IL-6 is an important cytokine in an inflammatory response and has the characteristics of promoting inflammation (58). TNF- $\alpha$ is a pro-inflammatory cytokine secreted by lymphoid $\mathrm{T}$ cells and plays an important role in various processes, such as inflammation and apoptosis $(59,60)$.

PQQ plays an important role in anti-inflammation, apoptosis, and autophagy. Therefore, this study investigated the effects of PQQ on inflammation, apoptosis, and autophagy in the rats SCI model. Besides, the effects of PQQ on apoptosis and inflammation of astrocytes induced by lipopolysaccharide (LPS) in vitro explore the neuroprotective effect of PQQ. We present the following article in accordance with the ARRIVE reporting checklist (available at https://dx.doi.org/10.21037/atm-21-1951).

\section{Methods}

\section{Grouping of experimental animals}

Adult male Sprague-Dawley rats (200-250 g) were obtained from the experimental Animal Center of Nantong University (Nantong, China). All animal care, breeding, and testing procedures were approved according to the Animal Care and Use Committee of Nantong University (license number: S20190920-003) and the Animal Care Ethics Committee of Jiangsu Province, in compliance with internationally recognized and institutional guidelines for the care and use of animals. A protocol was prepared before the study without registration.

All animals $(\mathrm{n}=60)$ were divided into four groups: Normal group, Sham group, SCI group, SCI + PQQ group (there were 15 rats in each group). At each stage of the experiment, the corresponding author Meijuan Yan was aware of the group allocation. The SCI (SCI) model was established at the 9th thoracic vertebra of rats using IH-0400 spinal cord striker (precision systems and instrumentation, PSI). The specific steps are as follows: Animals were given an intraperitoneal injection of compound anesthetics $(0.2-0.3 \mathrm{~mL} / 100 \mathrm{~g})$. After anesthesia, the back hair of rats was removed with a hair shaving device and disinfected with iodophor. Cut the skin above the thoracic vertebrae with a scalpel, separate the muscles above the spine and on 
both sides with ophthalmic scissors, expose the 8th thoracic vertebrae to the 10th thoracic vertebrae, and open the lamina with bite forceps in the 9th thoracic vertebrae to expose the spinal cord without damaging the dura.IH-0400 spinal cord striker with a $2.5 \mathrm{~mm}$ impact head was used to strike the spinal cord at the position of spinal cord exposure, and the striking intensity was $150 \mathrm{kDynes}$. Observe whether there is edema and congestion in the hitting area to preliminarily judge whether the establishment of the SCI model is successful, and then suture the wound with suture. During modeling and breeding, some rats may die, which were taken out and replaced with new rats for ensuring the same number of rats in each group. In the Sham group, only the lamina was opened without SCI. In the SCI + PQQ group, PQQ ( $5 \mathrm{mg} / \mathrm{kg} / \mathrm{d}$ ) was injected intraperitoneally $2 \mathrm{~h}$ in rats after anesthesia recovery. Then the rats were sent to the animal center of Nantong University $\left(18-26^{\circ} \mathrm{C}\right.$, free diet, weak light). After the establishment of the SCI model, we assisted the rats in urinating until they could urinate autonomously.

\section{Basso Beattie Bresnahan Scoring assay}

Basso, Beattie, and Bresnahan open field scale were used to evaluate the recovery of hind limb movement after SCI in rats. The score range was 0 (complete paralysis) to 21 (normal movement). According to the BBB rating scale, rats in each group were observed and scored at different time points after SCI.

\section{Hematoxylin and Eosin (HE) staining and Nissl staining}

The spinal cord tissues of each group were fixed with $4 \%$ paraformaldehyde at $4{ }^{\circ} \mathrm{C}$. The spinal cord tissue was placed at the bottom of a $50-\mathrm{mL}$ centrifuge tube and dehydrated in $10 \%, 20 \%$, and $30 \%$ sucrose solution. Twelve micrometers frozen sections were made and detected. Experiments were performed according to Hematoxylin and Eosin Staining Kit (Beyotime Biotechnology) and Nissl staining instructions (Beyotime Biotechnology).

\section{Western Blot analysis}

Cells and tissue samples were lysed with extraction buffer. The tissues were pulverized with an ultrasonic pulverizer, placed on ice for $30 \mathrm{~min}$, and then centrifuged at $12,000 \mathrm{r} / \mathrm{min}$ for $15 \mathrm{~min}$. The concentration of the protein was tested by using a Bicinchonininc acid (BCA) analysis kit (Beyotime, Jiangsu, China). Protein extracts were heat-denatured at $95^{\circ} \mathrm{C}$ for $5 \mathrm{~min}$, electrophoretically separated on Sodium dodecyl sulfate-polyacrylamide gel electrophoresis (SDS-PAGE), and transferred to polyvinylidene fluoride (PVDF) membrane (Millipore, Bedford, MA, USA) for $120 \mathrm{~min}$ at $100 \mathrm{~V}$. The membranes were blocked with $5 \%$ skim milk and then incubated at $4{ }^{\circ} \mathrm{C}$.

Overnight with Bcl-2 antibody [diluted 1:500 in tris aminomethane-hydrochloric acid buffer saline (TBS); Santa Cruz, Cell Signaling Technology], Rabbit-antiActive-Caspase3 (1:1,000, ab49822, Abcam), Rabbit-antiCaspase3 (1:1,000, ab184787, Abcam), Rabbit-anti-Bax (1:1,000, ab12503, Abcam), Rabbit-anti-Bcl2 (1:1,000, NB100-56101, NOVUS), Rabbit-anti-LC3 (1:500, ab128025, Abcam), Rabbit-anti-LAMP2 (1:1,500, L0668, Sigma), Rabbit-anti-phospho-Akt (p-AKT) (1:1,000, 8200S, Cell Signaling), Rabbit-anti-AKT (1:1,000, C67E7, Cell Signaling) and Mouse-anti- $\beta$-actin (1:5,000, A1978, Sigma). The PVDF membrane was washed with tris aminomethanehydrochloric acid buffer saline tween (TBST) (TBS with $0.1 \%$ Tween 20) for $10 \mathrm{~min}$ at least three times and followed by a reaction with the secondary antibody conjugated with goat anti-rabbit or goat anti-mouse horseradish peroxidase (HRP) dilution 1:1,000 (Santa Cruz) at room temperature for $2 \mathrm{~h}$. After the membrane was washed, the HRP activity was detected using Beyo enhanced chemiluminescent (ECL) Star (Beyotime, Jiangsu, China). The image was scanned with a GS800 Densitometer Scanner (Bio-Rad), and the data were analyzed using PDQuest 7.2.0 software (Bio-Rad).

\section{Enzyme-linked immunosorbent assay (ELISA)}

Spinal cord tissue samples were prepared or astrocytes were treated with or without $1 \mu \mathrm{g} / \mathrm{mL}$ LPS or/and $10 \mu \mathrm{g} / \mathrm{mL}$ for 3, 12, and $24 \mathrm{~h}$. Cell supernatants were collected, and the tissues were lysed by radio immunoprecipitation assay (RIPA) lysis buffer (Absin). The lysates were centrifuged at $12,000 \mathrm{r} / \mathrm{min}$ for $15 \mathrm{~min}$. Levels of TNF- $\alpha$, IL- 6 or IL-1 $\beta$ were assessed using the ELISA kits (BD Biosciences, R\&D Systems) according to the manufacturer's directions. Results were read using a 96-well plate reader (Biotek Synergy2) at a $450 \mathrm{~nm}$ wavelength.

\section{Tissue immunobistochemistry}

The spinal cord tissues of each group were fixed with $4 \%$ paraformaldehyde at $4{ }^{\circ} \mathrm{C}$. The spinal cord tissue was placed at the bottom of $50 \mathrm{~mL}$ centrifuge tube and dehydrated in 
$10 \%, 20 \%$, and $30 \%$ sucrose solution. Twelve micrometers frozen sections were made and detected. All sections were blocked with blocking solution [10\% goat serum, $3 \%$ bovine serum albumin (BSA), and $0.1 \%$ Triton $\mathrm{X}-100]$ for $1 \mathrm{~h}$ at $37^{\circ} \mathrm{C}$ and then incubated overnight at $4{ }^{\circ} \mathrm{C}$ with Rabbit-anti-Active-Caspase3 (1:500, ab49822, Abcam), Rabbit-anti-Caspase3 (1:500, ab184787, Abcam), Rabbitanti-LC3 (1:100, ab128025, Abcam), Rabbit-anti-LAMP2 (1:400, L0668, Sigma), Rabbit-anti-Bcl-2 (1:500, NB10056101, NOVUS) and Rabbit-anti-Bax (1:500, ab12503, Abcam); $0.01 \mathrm{M}$ phosphate buffer saline (PBS) was used to wash them for $10 \mathrm{~min}$ at 3 times and followed by incubating with a mixture of FITC- or Cy3-conjugated secondary antibodies for $2 \mathrm{~h}$ at room temperature and then being washed again with PBS for $10 \mathrm{~min}$ at 3 times. The stained sections were examined with a Leica fluorescence microscope (Leica DM 5000B, Germany).

\section{Primary astrocytes culture}

Pure astrocytes culture was prepared from 1-day-old Sprague-Dawley rats. The spinal cord was exposed after opening the spine, and the surface membrane of the spinal cord was removed. Then spinal cord was cut in tissue dissection solution (99\% PBS, $1 \%$ penicillin/streptomycin) and centrifuged at $1,200 \mathrm{r} / \mathrm{min}$ for $5 \mathrm{~min}$. Enzymatic hydrolysis with $0.25 \%$ trypsin (Gibco-BRL) at $37{ }^{\circ} \mathrm{C}$ for $15 \mathrm{~min}$. After the cells were incubated at $37^{\circ} \mathrm{C}$ and $5 \% \mathrm{CO}_{2}$ for $72 \mathrm{~h}$, the medium was replaced to remove cell debris and non-adhesion cells.

\section{Cell Counting Kit-8 (CCK-8) test}

Cells were seeded onto a 96-well plate and then treated with or without $1 \mu \mathrm{g} / \mathrm{mL}$ LPS for $12 \mathrm{~h}$. Subsequently, cells were incubated in a humidified atmosphere with $5 \% \mathrm{CO}_{2}$ at $37^{\circ} \mathrm{C}$ for $2 \mathrm{~h}$ after adding CCK- 8 test solution (Dojindo, Japan), and the cell proliferation ability was detected by using a microplate reader at $450 \mathrm{~nm}$.

\section{Statistical analysis}

All data were expressed as mean \pm standard deviation $(\bar{x} \pm s)$, using one-way analysis of variance (ANOVA, comparison between multiple groups) and Tukey multiple comparison processing (comparison between the two groups), With a $\mathrm{P}$ value $<0.05$ indicating statistical significance. All experiments in this subject were repeated at least three times under the same conditions. Drawing software and statistical analysis software are Image J, Adobe Photoshop 6.0, and GraphPad Prism 8.0. All experimental data of this study can be obtained from the Key Laboratory of Neuroregeneration and the Archives of Nantong University.

\section{Results}

\section{Effect of $P Q Q$ on pathological changes after SCI in rats}

In order to understand the pathological changes in the spinal cord after SCI in rats, four days after the establishment of the SCI model, The SCI + PQQ group was continuously administered for four days after SCI. The spinal cord tissue $0.5 \mathrm{~cm}$ above and below the injury center was taken for HE staining. After the establishment of the SCI model, compared with the Normal group and the Sham group, the cell infiltration was increased in the SCI group, and the nerve fibers and cells were disordered (Figure 1A).

To understand whether neurons changed in the process of SCI, Nissl staining was used to observe the changes of cell morphology in frozen sections. In the Normal group, the cell body and nucleus of neurons were observed clearly, the cell body was hypertrophy and the cytoplasm staining was obvious ( $\mathrm{m}$ in Figure 1B). The morphology of neurons in the Sham group was changed, the cell body was reduced and the cytoplasm staining was weakened (n in Figure 1B). After SCI, the number of cells increased and neurons decreased significantly (o in Figure 1B). In the SCI + PQQ group, the number of neurons increased, the cytoplasm staining increased, and the cell morphology was sparsely visible ( $\mathrm{p}$ in Figure 1B).

\section{Effect of $P Q Q$ on BBB score after SCI in rats}

To investigate whether PQQ can promote the recovery of motor function after SCI in rats, a rat model of SCI was established and randomly divided into Normal group, Sham group, SCI group, and SCI + PQQ group (continuous administration of $5 \mathrm{mg} / \mathrm{kg} / \mathrm{d}$ by intraperitoneal injection $2 \mathrm{~h}$ after SCI). The total cycle of the model was $28 \mathrm{~d}$. The BBB scores of rats in each group were detected at 4, 7, 14, 21, and 28 d. Compared with the Normal group and Sham group, the hind limb motor function of the SCI group and the SCI + PQQ group at each time point was significantly decreased $(\mathrm{P}<0.05)$; Although the scores of the SCI + PQQ group at $0,4,7,14,21$, and $28 \mathrm{~d}$ after SCI were significantly higher than SCI group, they were still lower than Normal 
A
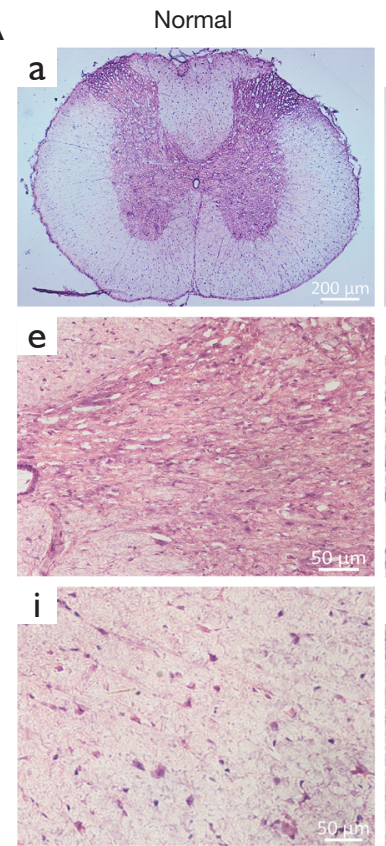

B
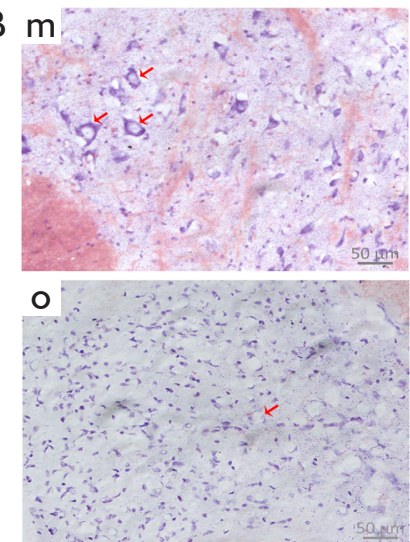

D

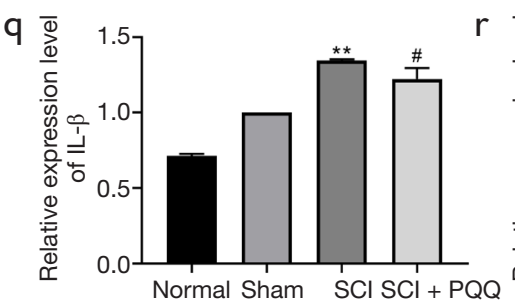

Sham
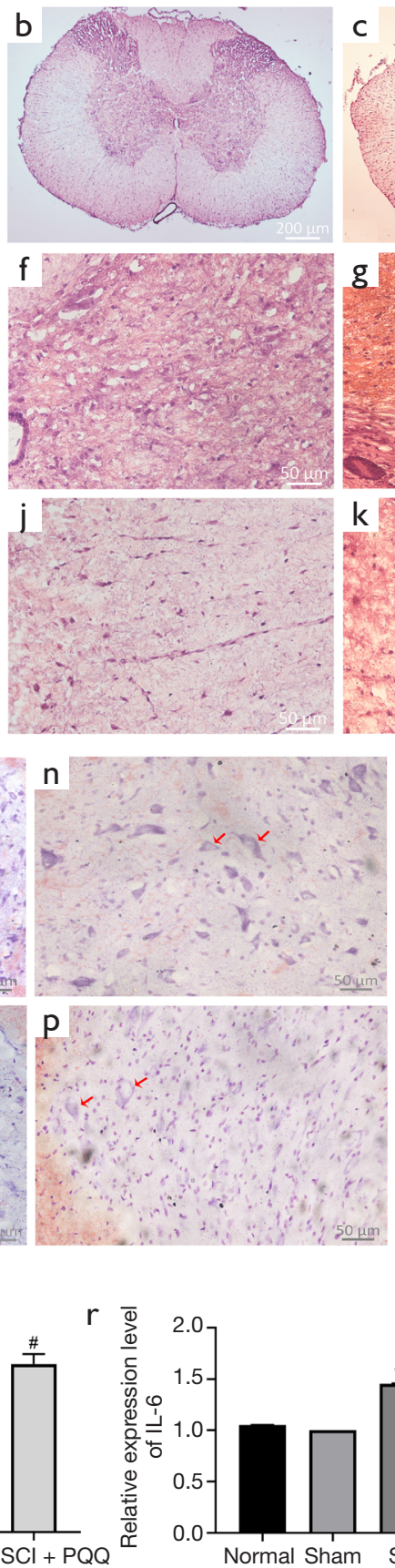

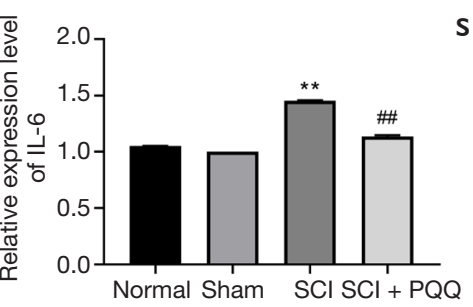

$\mathrm{SC}$
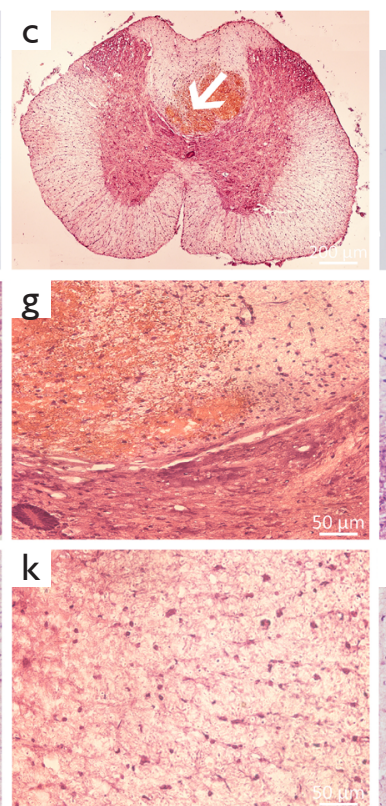

C

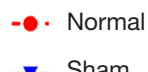

$-\mathrm{SCl}$

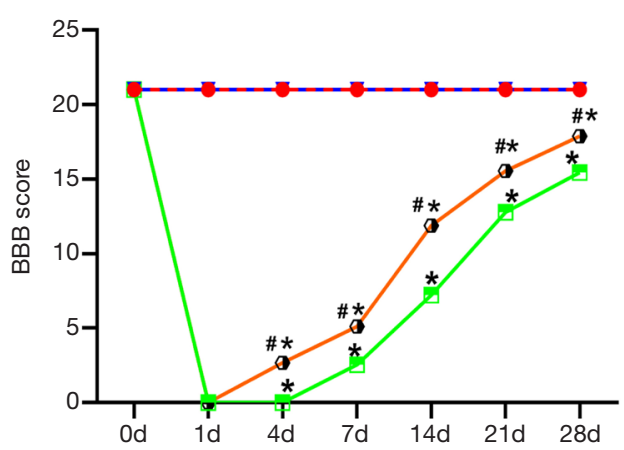

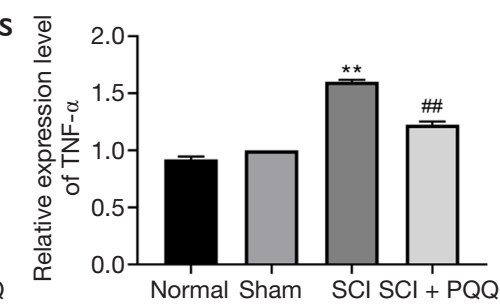

Figure 1 Pathological changes of spinal cord and BBB score in rats after SCI. (A) HE staining in spinal cord; (a,e,i): Normal group; (b,f,j): Sham group; $(\mathrm{c}, \mathrm{g}, \mathrm{k})$ : SCI group; (d,h,l): SCI + PQQ group; (e-h): periphery of the central canal in the spinal cord; (i-l): spinal cord white matter; In SCI + PQQ group, PQQ was injected intraperitoneally $2 \mathrm{~h}$ after recovery with a dose of $5 \mathrm{mg} / \mathrm{kg} / \mathrm{d}$, and saline was injected in the other three groups with the same method; After $4 \mathrm{~d}$ of continuous injection, samples were collected; In addition to BBB score, all experimental injection methods, doses and sampling time points were the same; The arrow shows the damaged part; bar $=200 \mu \mathrm{m}$ (a,b,c,d); Bar $=50 \mu \mathrm{m}$ (e-l). (B) Nissl staining; (m) Normal group; (n) Sham group; (c) SCI group; (d) SCI + PQQ group. bar =50 $\mu \mathrm{m}$. (m-p); The arrows represent the neuron cells. (C) BBB scores were compared at different time after SCI; The injection method and dose of each group were the same as the previous treatment; The only difference was that the experiment needed $28 \mathrm{~d}$ of continuous injection; ", $\mathrm{P}<0.05$ vs. SCI; *, $\mathrm{P}<0.05$ vs. Sham or Normal. (D) ELISA was used to detect the expression changes of pro-inflammatory cytokines (IL1- $\beta$, IL-6 and TNF- $\alpha$ ) after SCI in rats; **, $\mathrm{P}<0.01$ vs. Sham; ${ }^{\#}, \mathrm{P}<0.05$ vs. SCI; "\#, $\mathrm{P}<0.01$ vs. SCI. SCI, spinal cord injury; BBB, Basso Beattie Bresnahan; $\mathrm{PQQ}$, pyrrroloquinoline quinone; ELISA, enzyme-linked immunosorbent assay. 
group and Sham group, and the difference was statistically significant $(\mathrm{P}<0.05$, Figure 1C).

\section{Effect of PQQ on Bcl-2, Bax after SCI in rats}

$\mathrm{Bcl}-2$, Bax are proteins related to apoptosis. The expression changes of Bcl-2, Bax, and Bcl-2 / Bax ratio after SCI in each group were detected by Western Blot. After SCI, compared with the Normal group and Sham group, the expression level of Bax in the SCI group was significantly increased $(\mathrm{P}<0.05)$, the expression level of $\mathrm{Bcl}-2$ was significantly decreased $(\mathrm{P}<0.05)$, and the ratio of $\mathrm{Bcl}-2 / \mathrm{Bax}$ was significantly decreased $(\mathrm{P}<0.05)$. In the $\mathrm{SCI}+\mathrm{PQQ}$ group, the protein expression level of Bax was decreased $(\mathrm{P}<0.05)$, the protein expression level of $\mathrm{Bcl}-2$ was increased $(\mathrm{P}<0.05)$, and the ratio of $\mathrm{Bcl}-2 / \mathrm{Bax}$ was significantly increased $(\mathrm{P}<0.05$, Figure $2 A-2 D)$.

Western Blot results showed that the expression level of anti-apoptotic protein Bcl-2 changed. To observe the localization of $\mathrm{Bcl}-2$ in neurons after SCI, the localization of $\mathrm{Bcl}-2$ in neurons was detected by tissue immunofluorescence. In the Sham group, the positive signals of Bcl-2 and NeuN were evenly distributed and there was a large number of co-localization (Figure 2E). Compared with the Sham group, the positive signals of Bcl-2 and NeuN in the SCI group were weakened, and there was few co-location (Figure 2F). Compared with the SCI group, the positive signal of NeuN in the SCI + PQQ group was significantly increased, and there was a large number of colocalization between Bcl-2 and NeuN (Figure 2G).

\section{Effect of PQQ on Active-Caspase3/Caspase3 protein expression after $S C I$ in rats}

Apoptosis is a cascade reaction of proteases, apoptosis signal can induce the activation of apoptosis marker Caspase 3 and degrade intracellular proteins to death. Caspase 3 can be used as a downstream regulatory mechanism of $\mathrm{Bcl}-2$ and Bax. Bcl-2 can be used as a direct substrate of Caspase3, cleavage into pro-apoptotic fragments. In this study, the expression of Active-Caspase 3 after SCI was detected by Western Blot. The expression of Active-Caspase3 in the SCI group was significantly higher than that in the Normal group and Sham group $(\mathrm{P}<0.05)$. After PQQ treatment, the expression level was higher than that in the Normal group and Sham group. However, compared with the SCI group, the expression level was decreased obviously, the difference was statistically significant $(\mathrm{P}<0.05$, Figure $3 A, 3 B)$.

\section{Expression of Active-Caspase 3 and Caspase 3 in neurons after $S C I$ in rats treated by $P Q Q$}

To further observe the localization of Active-Caspase 3 and Caspase 3 in neurons, double immunofluorescence staining was used. Active-Caspase 3 is mainly located in the cytoplasm of neurons in the Normal group (Figure 4A), expressed in the nucleus and cytoplasm of neurons in the Sham group (Figure 4B), mainly localized in the nucleus in the SCI group (Figure 4C), and expressed in the nucleus and cytoplasm of neurons in the SCI + PQQ group (Figure 4D).

Caspase 3 was mainly located in the cytoplasm of neurons in the Normal group and Sham group (Figure 4E, $4 F$ ), distributed in the nucleus of the SCI group (Figure $4 G$ ), and expressed in the nucleus and cytoplasm of neurons in the SCI + PQQ group (Figure 4H).

\section{Expression of Active-Caspase 3 and Caspase 3 in GFAP positive astrocytes after $S C I$ in rats treated by $P Q Q$}

It has been reported that astrocytes after SCI may undergo apoptosis $(33,61)$. To further observe the localization of Active-Caspase 3 and Caspase 3 in glial fibrillary acidic protein (GFAP) positive astrocytes, the frozen sections of the spinal cord were stained by immunofluorescence. Active-Caspase3 was mainly located in the cytoplasm of astrocytes in the Normal group (Figure $5 A$ ) and was expressed in the nucleus and cytoplasm of astrocytes in the Sham group (Figure 5B). In the SCI group, Active-Caspase3 was mainly located in the nucleus (Figure 5C). After PQQ treatment, Active-Caspase 3 was expressed in the nucleus and cytoplasm of astrocytes (Figure 5D).

Immunofluorescence double-labeling results showed that Caspase 3 was mainly localized in the cytoplasm of astrocytes in the Normal group and Sham group (Figure $5 E, 5 F$ ), and was mainly localized in the nucleus in the SCI group (Figure 5G). After PQQ treatment, Active-Caspase3 was expressed in the nucleus and cytoplasm of astrocytes (Figure 5 H).

\section{Effect of PQQ on LC3 after SCI in rats}

Autophagy has two-way functions of inhibiting apoptosis and activating apoptosis, and LC3a/b is a marker of autophagy. Therefore, in this study, Western Blot was used to detect the expression of LC3a/b in spinal cord tissue of rats after SCI. Compared with the Normal group and Sham group, the expression level of LC3 in the SCI group was significantly increased, and the difference was statistically significant 
A

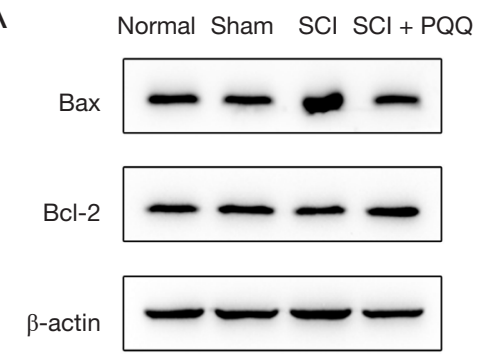

C

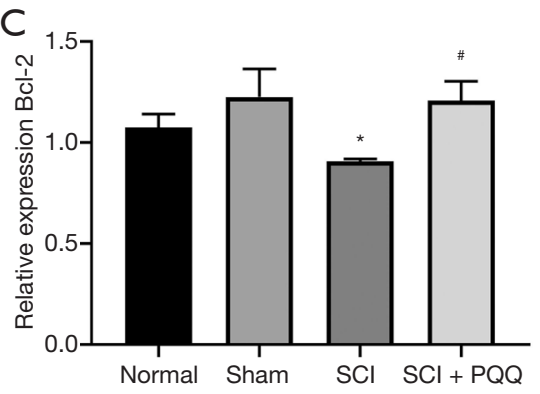

B

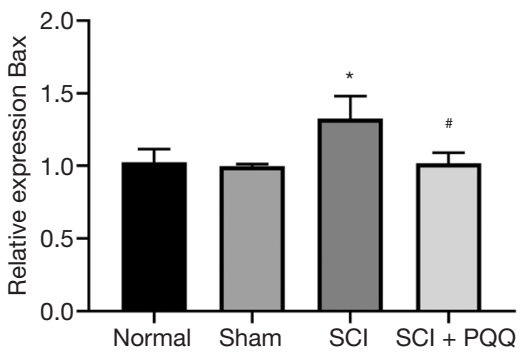

$\mathrm{D}$

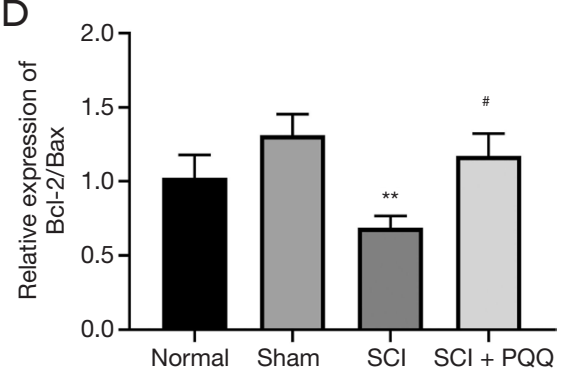

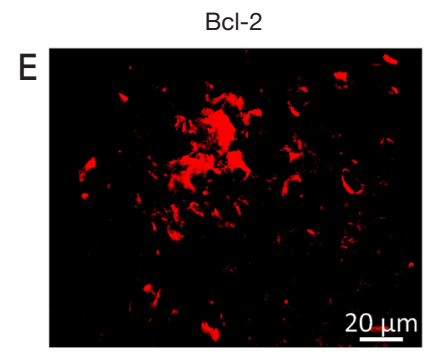
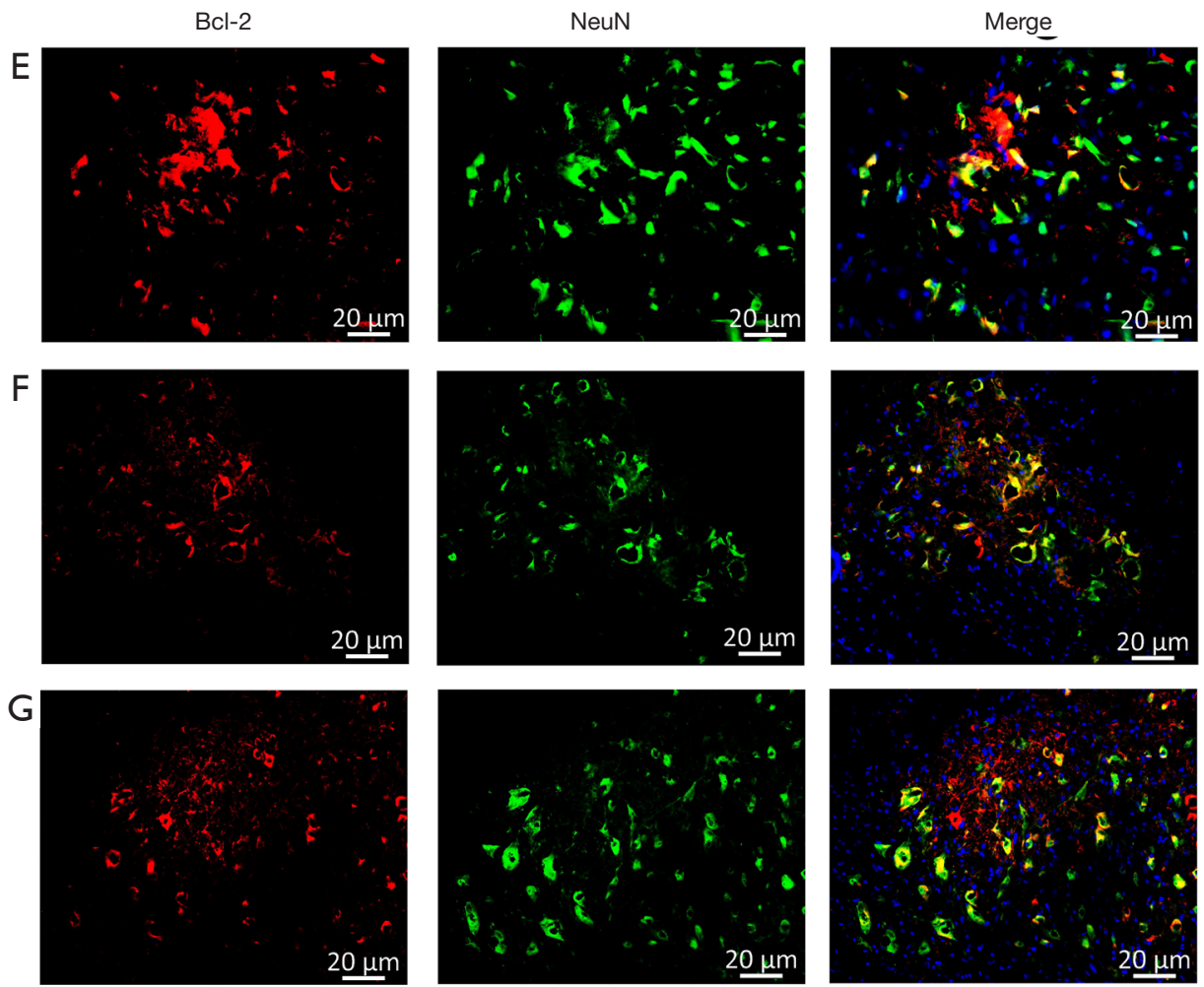

Figure 2 Expression change of Bcl-2, Bax and their co-localization with neurons and astrocytes after SCI. (A) Western Blot detect the expression Bcl-2 and Bax; (B) Statistical diagram of Bax/ $\beta$-actin; (C) Statistical diagram of Bcl-2/ $\beta$-actin; (D) Statistical diagram of Bcl-2/Bax; *, $\mathrm{P}<0.05$ vs. Sham; **, $\mathrm{P}<0.01$ vs. Sham; ;, $\mathrm{P}<0.05$ vs. SCI; (E-G) Double immunofluorescence of Bcl-2 and neurons in spinal cord; (E) Sham group; (F) SCI group; (G) SCI + PQQ group. Bcl-2 (Red); NeuN (Green); Hoechst (Blue). SCI, spinal cord injury; PQQ, pyrrroloquinoline quinone. 

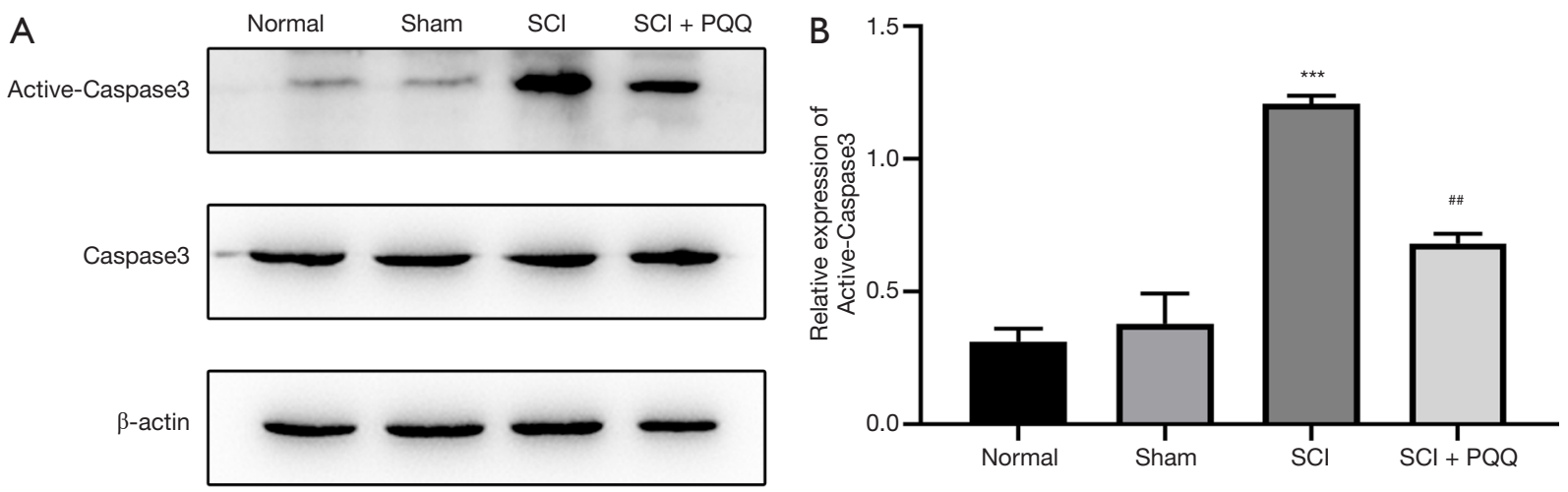

Figure 3 Expression of Active-Caspase3/Caspase3 after SCI by Western Blot. (A) Western Blot detect the expression of Active-Caspase3 protein; (B) the statistical figure of Active-Caspase3/Caspase3. *** ${ }^{*}<0.001$ vs. Sham; ${ }^{\# \#}, \mathrm{P}<0.05$ vs. SCI. SCI, spinal cord injury; PQQ, pyrrroloquinoline quinone.

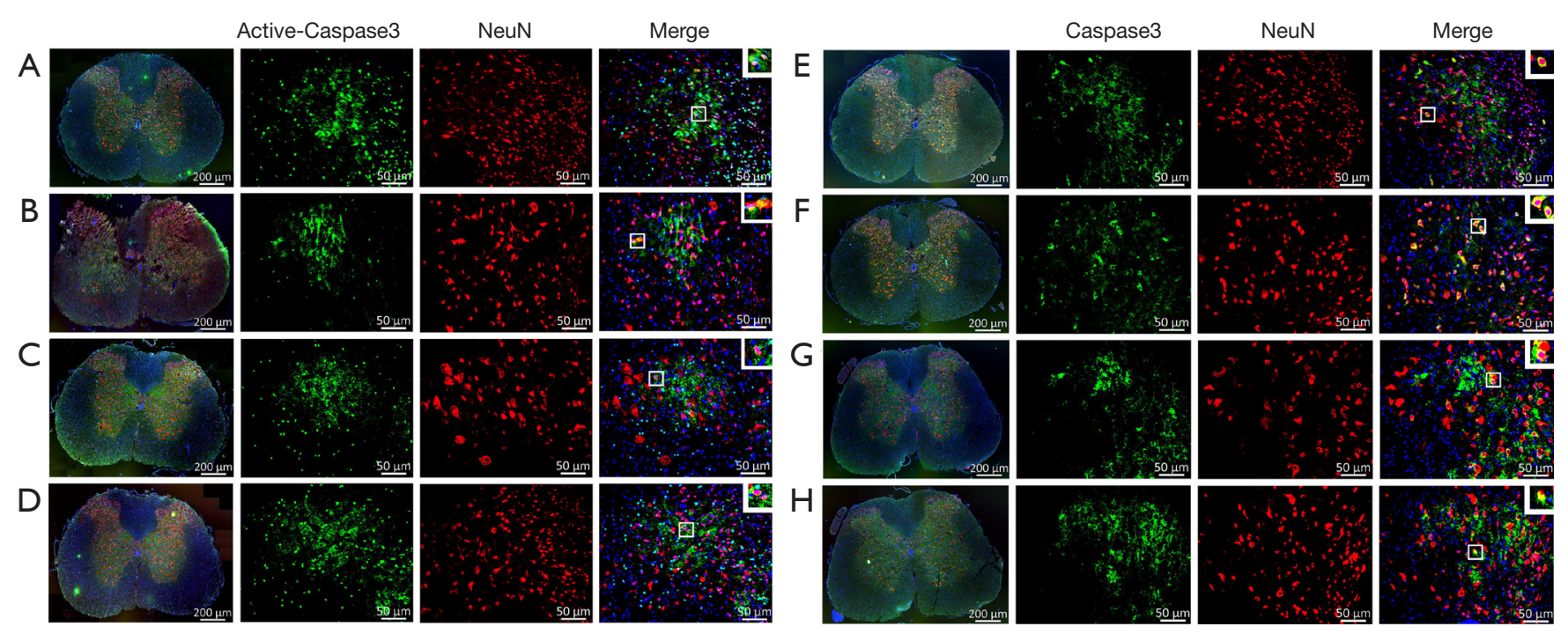

Figure 4 Double immunofluorescence of Active-Caspase3 and Caspase3 with NeuNin spinal cord Expression of Active-Caspase3 in Neurons of Rats after SCI; (A) Normal group; (B) Sham group; (C) SCI group; (D) SCI + PQQ group. Active-Caspase3 (Green); NeuN (Red); Hoechst (Blue); bar $=200 \mu \mathrm{m}$; Bar $=50 \mu \mathrm{m}$. Expression of Caspase3 in Neurons of Rats after SCI; (E) Normal group; (F) Sham group; (G) SCI group; (H) SCI + PQQ group. Caspase3 (Green); NeuN (Red); Hoechst (Blue); bar =200 $\mu \mathrm{m}$; Bar =50 $\mu \mathrm{m}$. SCI, spinal cord injury; PQQ, pyrroloquinoline quinone.

$(\mathrm{P}<0.05)$; Compared with the SCI group, the expression level of the SCI + PQQ group was increased, but the difference was not statistically significant $(\mathrm{P}>0.05$, Figure $6 A, 6 B)$.

Western Blot results showed that the expression level of autophagy marker LC3a/b changed. In order to observe the localization of LC3 in astrocytes after SCI, the expression of LC3 in astrocytes was detected by immunofluorescence double labeling. LC3 was expressed in the cytoplasm and nucleus of GFAP positive cells; Compared with the Normal group (Figure 6C) and the Sham group (Figure 6D), the colocalization of LC3 and GFAP positive cells in the SCI group was decreased (Figure 6E); compared with SCI group, the co-localization of LC3 and GFAP positive cells was increased in the SCI + PQQ group (Figure $6 F$ ). 


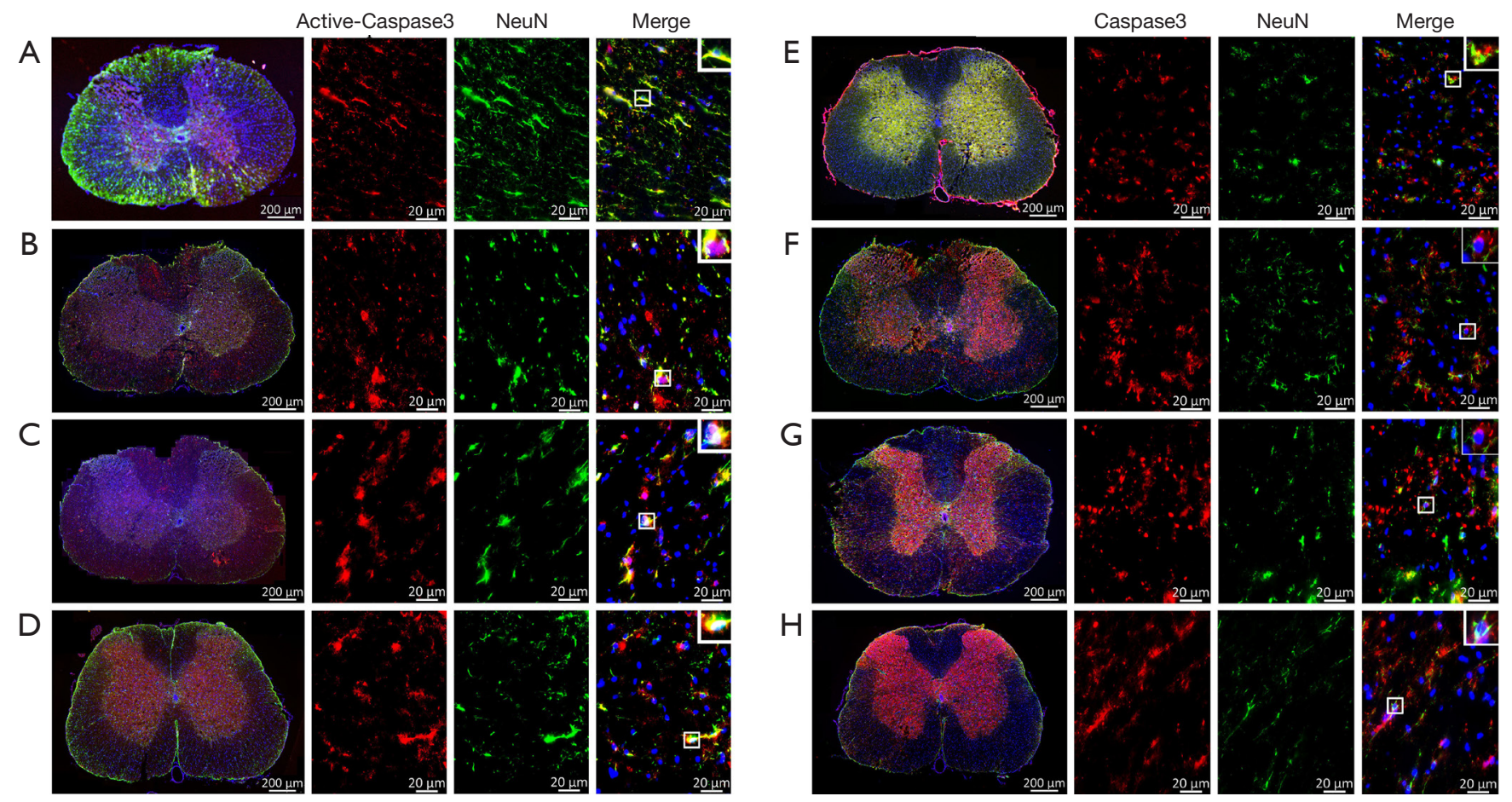

Figure 5 Double immunofluorescence of Active-Caspase3 and Caspase3 with GFAP in spinal cord. Expression of Active-Caspase3 in GFAP positive astrocytes of Rats after SCI; (A) Normal group; (B) Sham group; (C) SCI group; (D) SCI + PQQ group. Active-Caspase3 (Red); GFAP (Green); Hoechst (Blue); bar $=200 \mu \mathrm{m}$; Bar $=50 \mu \mathrm{m}$. Expression of Caspase3 in Neurons of Rats after SCI; (E) Normal group; (F) Sham group; (G) SCI group; (H) SCI + PQQ group. Caspase3 (Red); GFAP (Green); Hoechst (Blue); bar =200 $\mu \mathrm{m}$; Bar =50 $\mu \mathrm{m}$. SCI, spinal cord injury; PQQ, pyrrroloquinoline quinone; GFAP, glial fibrillary acidic protein.

\section{Effect of PQQ on LAMP2 and p-AKT protein expression after $S C I$ in rats}

LAMP2 is a lysosomal marker interacting with autophagosomes. Since the expression level of autophagy marker LC3 changes during SCI, the expression level of LAMP2 in SCI tissues was detected by Western Blot. Compared with the Normal group and Sham group, the protein expression level of LAMP2 was up-regulated after SCI $(\mathrm{P}<0.05)$. After PQQ treatment, although the expression level of LAMP2 was higher than that in the Normal group and Sham group, the expression level of LAMP2 was lower than that in the SCI group, and the difference was statistically significant $(\mathrm{P}<0.05$, Figure $7 A, B)$.

Phosphatidylinositol 3 kinase (PI3 K)-AKT is an important anti-apoptotic signaling pathway in vivo. When apoptosis occurs, P13K can activate AKT, phosphorylate it, indirectly or directly help cells survive. Western Blot was used to detect the level of phosphorylated AKT (p-AKT). The results showed that compared with the Normal group and Sham group, the expression level of p-AKT was significantly increased after SCI $(\mathrm{P}<0.05)$. The expression level of the SCI + PQQ group was higher than that in the Normal group and Sham group, but the expression level of p-AKT was significantly lower than that in the SCI group, and the difference was statistically significant $(\mathrm{P}<0.05$, Figure $7 A, C$ ).

\section{Effect of PQQ on primary astrocytes treated with LPS}

PQQ has a corresponding effect on an inflammatory response, apoptosis, and autophagy SCI model of rats. Some studies have shown that astrocytes will also undergo apoptosis after SCI $(33,62)$. In order to observe whether PQQ affects these processes in primary cultured astrocytes, the purity of primary cultured astrocytes was identified. The results showed that the purity of astrocytes was above 95\% (Figure $8 A-C$ ), which could be used for subsequent experiments.

In order to select the appropriate concentration of 


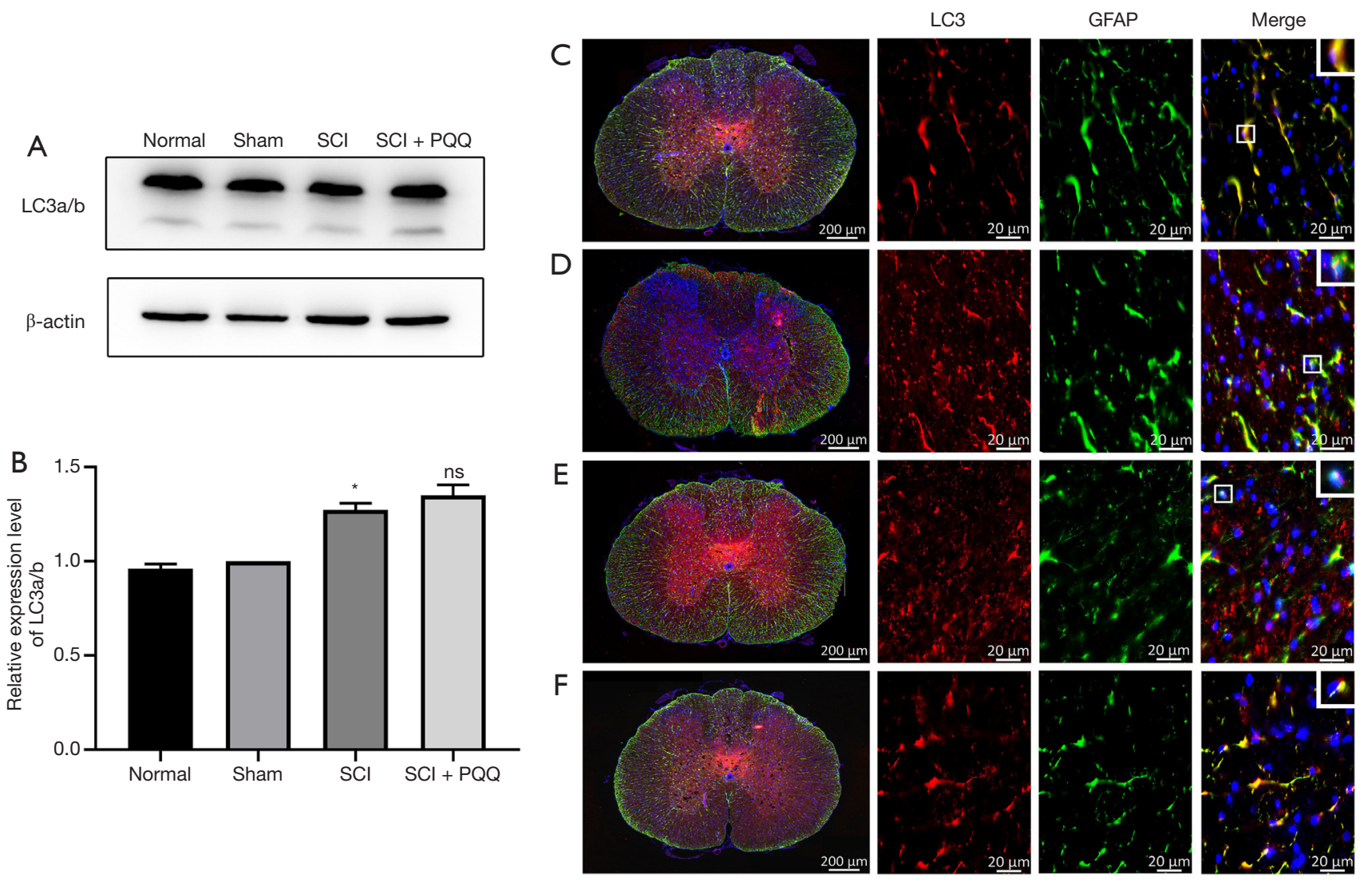

Figure 6 Expression and localization of LC3 in spinal cord. (A) Western Blot detect the LC3a/b expression; (B) Statistical diagram of LC3a/ b; *, P<0.05 vs. Sham; ns, >0.05 vs. SCI. (C,D,E,F) Double immunofluorescence of LC3 with GFAP. (C) Normal group; (D) Sham group; (E) SCI group; (F) SCI + PQQ group. LC3 (Red); GFAP (Green); Hoechst (Blue); bar =200 $\mu \mathrm{m}$; bar =20 $\mu \mathrm{m}$. LC3, microtubule associated protein 1 light chain 3; SCI, spinal cord injury; PQQ, pyrroloquinoline quinone; GFAP, glial fibrillary acidic protein.
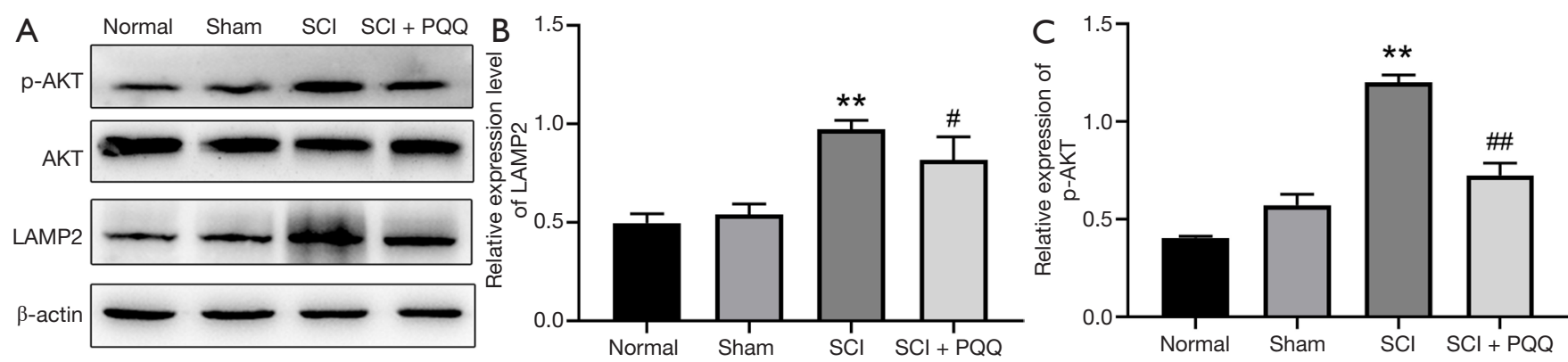

Figure 7 Expression of LAMP2 and p-AKT after SCI by Western Blot. (A) Western Blot detect the expression of LAMP2 and p-AKT; (B) statistical diagram of LAMP2/ $\beta$-actin expression, ${ }^{* *}, \mathrm{P}<0.01$ vs. Sham;, $\mathrm{P}<0.05$ vs. SCI; (C) statistical diagram of $\mathrm{p}$-AKT/ $\beta$-actin expression, **, $\mathrm{P}<0.01$ vs. Sham; ${ }^{\#}, \mathrm{P}<0.01$ vs. SCI. LAMP2, lysosomal associated membrane protein 2; SCI, spinal cord injury; PQQ, pyrrroloquinoline quinone. 


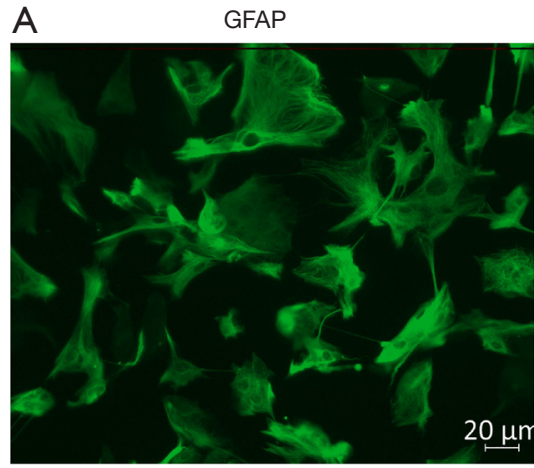

B
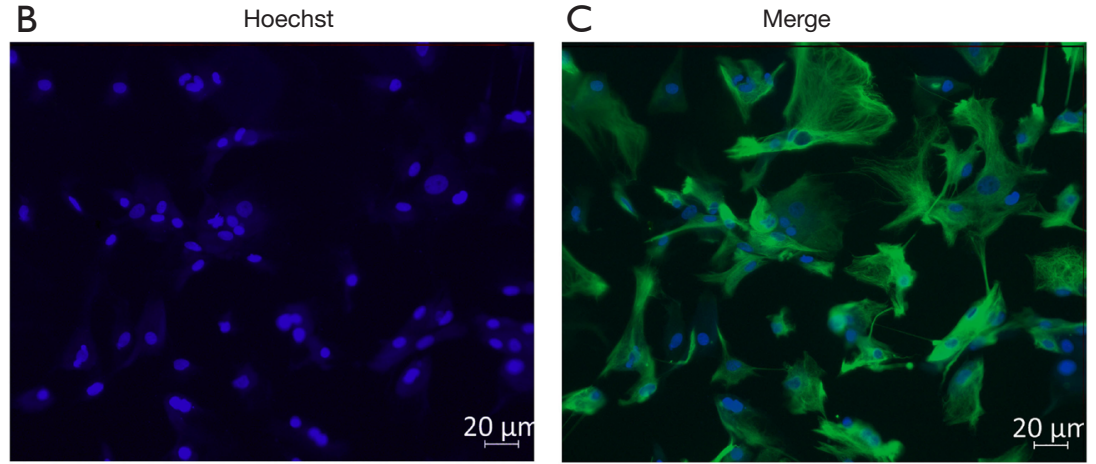

E

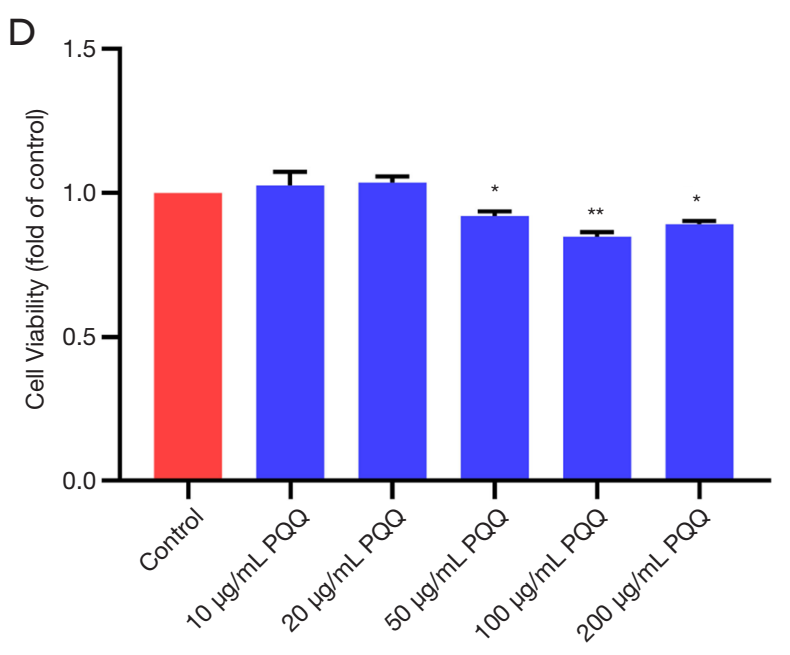

Active-Caspase3
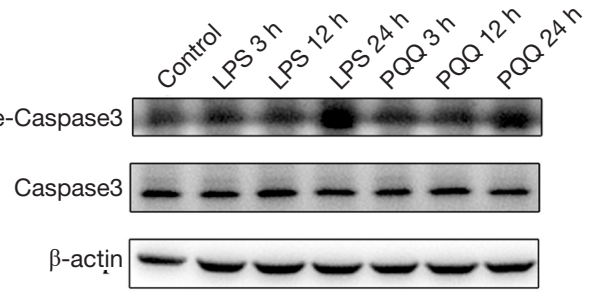

$F$
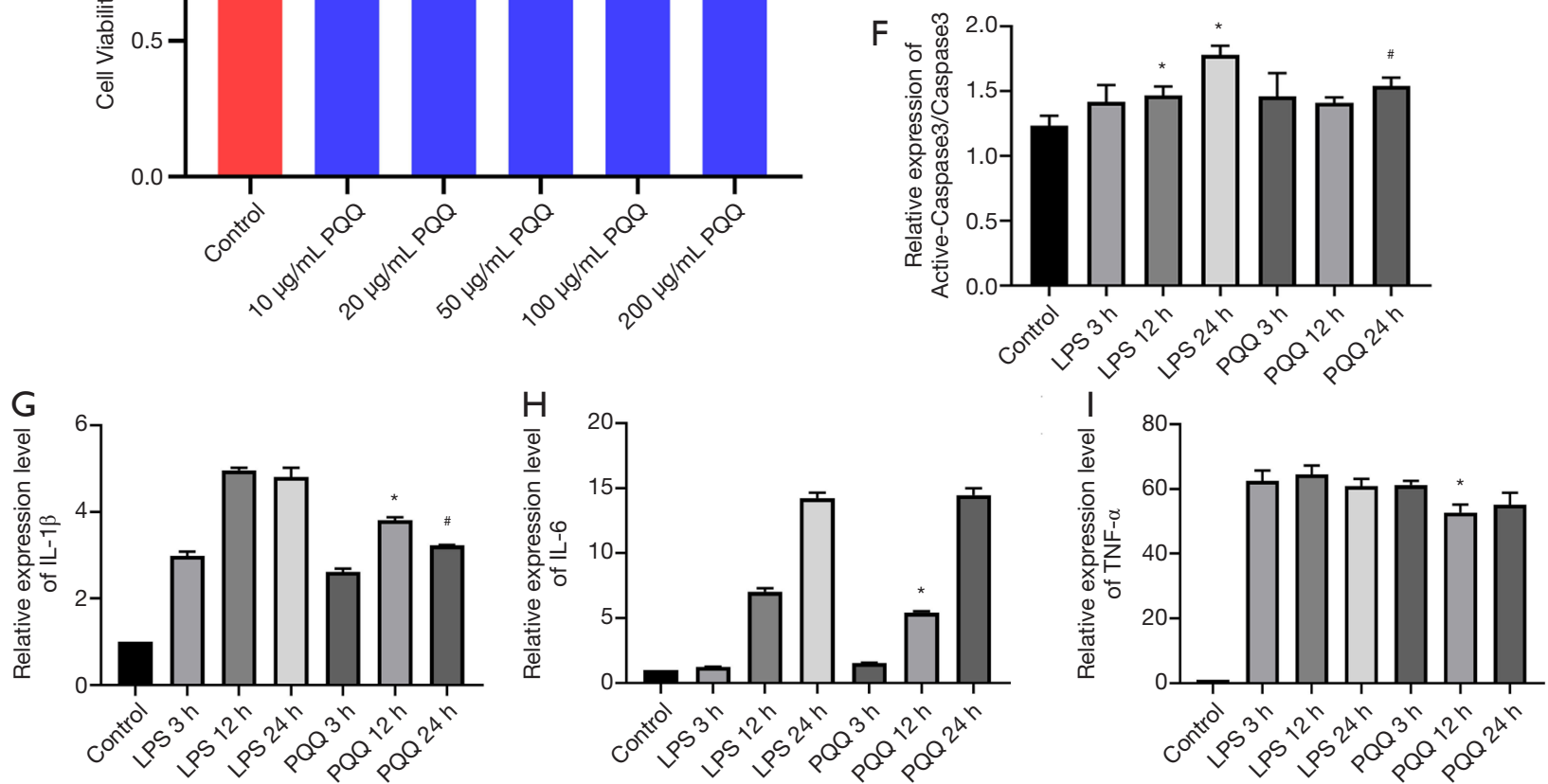

Figure 8 Effect of PQQ on LPS-induced astrocytes. Immunofluorescence staining was used to identify the purity of primary astrocytes, GFAP (Green, A); Hoechst (Blue, B); Merge of A and B (C), bar =20 $\mu \mathrm{m}$. (D) Detection of primary astrocyte activity by CCK-8; *, $\mathrm{P}<0.05$ vs. Control; **, $\mathrm{P}<0.01$ vs. Control; (E) Western Blot was used to detect the protein expression changes of Active-Caspase 3 inprimary astrocytes; (F) Statistical diagram of Active-Caspase3; * $\mathrm{P}<0.05$ vs. LPS $12 \mathrm{~h}$; * $\mathrm{P}<0.05$ vs. LPS 24 h; (G-I) The expression of pro-inflammatory cytokines IL-1- $\beta$, IL-6 and TNF- $\alpha$ in astrocytes were detected by ELISA; *, P<0.05 vs. LPS 12 h; *, P<0.05 vs. LPS 24 h. Control: Normal group without any treatment; LPS $3 \mathrm{~h}$ : astrocytes were treated with $1 \mu \mathrm{g} / \mathrm{mL}$ LPS for 3 h; LPS 12 h: astrocytes were treated with $1 \mu \mathrm{g} / \mathrm{mL}$ LPS for $12 \mathrm{~h}$; LPS $24 \mathrm{~h}$ : astrocytes were treated with $1 \mu \mathrm{g} / \mathrm{mL}$ LPS for $12 \mathrm{~h}$; PQQ $3 \mathrm{~h}$ : astrocytes were treated with $1 \mu \mathrm{g} / \mathrm{mL}$ LPS $+10 \mu \mathrm{g} / \mathrm{mL}$ PQQ for $3 \mathrm{~h}$. PQQ $12 \mathrm{~h}$ : astrocytes were treated with $1 \mu \mathrm{g} / \mathrm{mL} \mathrm{LPS}+10 \mu \mathrm{g} / \mathrm{mL}$ PQQ for $12 \mathrm{~h}$; PQQ $24 \mathrm{~h}$ : astrocytes were treated with $1 \mu \mathrm{g} / \mathrm{mL}$ LPS $+10 \mu \mathrm{g} / \mathrm{mL}$ PQQ for $24 \mathrm{~h}$. PQQ, pyrrroloquinoline quinone; LPS, lipopolysaccharide; ELISA, enzyme-linked immunosorbent assay. 
PQQ, CCK-8 was used to detect the effect of different concentrations of PQQ $(0,10,20,50,100,200 \mu \mathrm{g} / \mathrm{mL})$ on the viability of astrocytes after $24 \mathrm{~h}$. The normal group without any treatment was set as the control group. The results showed that when the concentration of PQQ was greater than or equal to $50 \mu \mathrm{g} / \mathrm{mL}$, the viability of astrocytes was inhibited, and the difference was statistically significant $(\mathrm{P}<0.05$, Figure $8 D)$. It showed that when the concentration of PQQ was too high, it would have cytotoxicity. Therefore, $10 \mu \mathrm{g} / \mathrm{mL}$ PQQ was selected for subsequent experiments.

It has been reported that LPS can induce cell apoptosis. In this study, $1 \mu \mathrm{g} / \mathrm{mL}$ LPS was used to treat astrocytes to obtain the apoptosis model of astrocytes. The protein expression level of Active-Caspase3 in astrocytes of each group was detected by Western Blot. The results showed that there was no significant difference in the protein expression of Active-Caspase3 between the PQQ treatment group and the LPS treatment group at $3 \mathrm{~h}(\mathrm{P}>0.05)$. At $12 \mathrm{~h}$, the protein expression of Active-Caspase 3 in the PQQ treatment group was not significantly different from that in the LPS treatment group $(\mathrm{P}>0.05)$. At $24 \mathrm{~h}$, compared with the LPS treatment group, the expression of Active-Caspase3 in PQQ the treatment group decreased, the difference was statistically significant $(\mathrm{P}<0.05$, Figure $8 E, 8 F)$.

It has been reported that LPS can induce the inflammatory model of cells. In this study, astrocytes were treated with $1 \mu \mathrm{g} / \mathrm{mL}$ LPS to obtain the inflammatory model of astrocytes. The following experiments were carried out on this model. The expression levels of pro-inflammatory cytokines IL- $1 \beta$, IL-6, and TNF- $\alpha$ in astrocytes were detected by an ELISA kit. The results showed that there was no significant difference in IL-1 $\beta$, IL-6, and TNF- $\alpha$ between the PQQ treatment group and the LPS treatment group at $3 \mathrm{~h}(\mathrm{P}>0.05)$. Compared with the LPS treatment group for $3 \mathrm{~h}$, the expression levels of IL-1 $\beta$, IL-6, and TNF- $\alpha$ in the PQQ treatment group were significantly decreased $(\mathrm{P}<0.05)$. At $24 \mathrm{~h}$, compared with the LPS treatment group, the expression level of IL- $1 \beta$ in the PQQ treatment group was decreased, and the differences were statistically significant $(\mathrm{P}<0.05)$. There was no significant difference in the expression of IL- 6 and TNF- $\alpha(P>0.05$, Figure 8G-8I).

\section{Discussion}

PQQ is a small molecule aromatic tricyclic o-quinone compound that is ubiquitous in organisms. It is a new redox coenzyme and was first discovered as a dehydrogenase cofactor in 1964 (62). Although PQQ is widespread, there is still no evidence to prove that it can be synthesized by organisms themselves, and external intake seems to be the only source of mammals at present. The lack of PQQ will cause immune disorders, growth and reproduction disorders in mammals (11). The role of PQQ in cultured cells and animal models involves the activation and expression of nutritional factors, such as cAMP response elements, mitochondria, and peroxisome proliferator-activated receptors $(17,18)$. These co-activators and transcription factors can regulate the biological origin of mitochondria and cell energy metabolism (17-20).

SCI is a life-threatening degenerative disease, which usually causes sensory abnormalities, motor dysfunction, and even paralysis $(63,64)$. The results of this study showed that the BBB score decreased after SCI in rats, and the score increased significantly after PQQ treatment, which was consistent with the results of PQQ improving the complete motor paralysis function of rats' hind limbs after spinal cord hemisection (65), suggesting that PQQ has the effect of protecting the motor function of rats' hind limbs after SCI. However, the BBB score after PQQ treatment was still lower than Normal group and Sham group at each time point, and the differences were statistically significant. These results suggest that PQQ can improve the motor dysfunction of hind limbs after SCI at the time point of this experimental study, but it cannot completely cure SCI.

After the initial mechanical injury of the spinal cord, macrophages and monocytes were recruited in the injured site (64), neurons and glial cells changed destructively, and neurons around the injured site were lost $(66,67)$. In this study, the results of HE staining showed that the pathological changes of cell aggregation, congestion, and disorder of cell arrangement appeared in the injured site after SCI, which was significantly improved after PQQ treatment. The results of Nissl staining showed that the cell body and nucleus of neurons could be observed clearly in the Normal group, and the cell body of neurons was large and the staining was clear. In the Sham group, the cell body of neurons was slightly decreased and the staining became shallow, which might be due to the pathological changes of spinal cord tissue caused by stimulation such as stripping the spinal canal and exposing the spinal cord during the Sham group. After SCI, the number of neurons decreased, and the cell morphology of neurons could not be observed clearly. After PQQ treatment, the number of neurons increased, and the cell morphology of neurons was rare. These results indicated that PQQ improved cell aggregation and 
decreased the number of neurons.

Mechanical injury of the spinal cord causes strong inflammatory immune response, which will further lead to secondary injury (68). Some studies suggest that melatonin can exert its neuroprotective effect by inhibiting the inflammatory response after SCI (69). In this study, the expression levels of pro-inflammatory cytokines IL- $1 \beta$, IL-6, and TNF- $\alpha$ were significantly increased after SCI, and the expression level was significantly decreased after PQQ treatment, suggesting that PQQ and melatonin have similar inhibitory effects on the inflammatory response in SCI. Studies have shown that PQQ can down-regulate the overexpression of TNF- $\alpha$, IL- $1 \beta$, IL-6, monocyte chemoattractant protein-1 (MCP-1), and macrophage inflammatory protein-1 alpha (MIP-1 $\alpha)$ mRNA in LPSinduced microglia (70). In CNS, besides microglia, astrocytes also play an important role as neuroimmune cells. Astrocytes are the largest number of cells in the CNS, which plays a key role in maintaining the blood-brain barrier, regulating local blood flow, providing nutrition for neurons, regulating synapses and the inflammatory response (71-73). However, the role of PQQ in the inflammatory response of astrocytes has not been reported. In this study, astrocytes were induced by LPS and treated with PQQ to observe the effect of astrocytes on the inflammatory response. The results showed that PQQ treatment could reduce the expression of pro-inflammatory cytokines IL-1 $\beta$, IL-6, and TNF- $\alpha$ in LPS-induced astrocytes, which is similar to the role of PQQ in microglial inflammation (70), indicating that PQQ treatment can inhibit LPS-induced inflammation of astrocytes. In conclusion, PQQ inhibits the inflammatory response after SCI by inhibiting the inflammatory response of microglia and astrocytes.

It has been reported that apoptosis occurs immediately after SCI in rats and reaches its peak on the 7 th day in the literature (33). In CNS, apoptosis is closely related to autophagy. Autophagy plays a neuroprotective role $(40,42)$. It can promote cell survival by removing damaged mitochondria and harmful proteins in the body (43), and apoptosis is more likely to occur after autophagy imbalance $(74,75)$. The balance between autophagy and apoptosis plays a crucial role in maintaining cell life, and members of the Bcl-2 family also play a certain role in maintaining the balance between autophagy and apoptosis (76). Therefore, the effects of SCI in rats and LPS-induced astrocytes on apoptosis and autophagy were considered. Studies have shown that brain injury can promote cell apoptosis, and PQQ can alleviate cell apoptosis, and it also has effects on the autophagy-related marker LC3 and lysosomal marker LAMP2 (77). In this study, the protein expression of apoptosis-related protein Bcl-2/Bax decreased after SCI, and the protein expression of its downstream apoptosis marker Active-Caspase3 increased. PQQ treatment could up-regulate the expression of $\mathrm{Bcl}-2 / \mathrm{Bax}$ and down-regulate the expression of Active-Caspase3, suggesting that PQQ can inhibit the apoptosis of the injured spinal cord. So the question "are neurons involved in PQQ inhibiting apoptosis after SCI?” was pointed out. In the present study, immunofluorescence double-labeling was used to observe the localization of Bcl-2, Active-Caspase3, Caspase3, and neuron marker NeuN. The results showed that the positive signals of Bcl-2 and NeuN were significantly decreased after SCI, and the positive signals of NeuN were significantly increased after PQQ treatment, and a large number of Bcl-2 and NeuN positive cells were co-located. After SCI, the localization of Active-Caspase3, Caspase3, and NeuN positive cells was transferred from cytoplasm to nucleus, which was reversed by PQQ treatment, suggesting that neurons were involved in the process of PQQ inhibiting apoptosis after SCI. Astrocytes have also been reported to undergo apoptosis during SCI $(33,61)$. Therefore, this study was further observed the co-localization of Active-Caspase3 and Caspase 3 with astrocyte marker GFAP, which is similar to the localization of Active-Caspase 3 and Caspase 3 with neurons, suggesting that besides neurons, astrocytes also play a role in PQQ inhibiting apoptosis after SCI. Based on these results, this study used Western Blot to observe the changes in the protein expression level of Active-Caspase3 in the LPS-induced astrocytes. PQQ treatment can downregulate the protein expression of Active-Caspase 3 in the LPS-induced astrocytes apoptosis model, and play its role in inhibiting apoptosis in the LPS-induced astrocytes apoptosis model. These results suggest that neurons and astrocytes are involved in the process of PQQ affecting apoptosis. The autophagy-lysosomal pathway plays an important role in clearing aggregated proteins and preventing cellular stress and neurodegenerative diseases (78).

Due to the close relationship between autophagy and apoptosis, the protein expression levels of LC3 and LAMP2 were observed in this study. The results showed that the expression level of LC3 increased after SCI and continued to increase after PQQ treatment, but the difference was not statistically significant. LAMP2 expression increased after SCI and was down-regulated by PQQ treatment. in order to further observe the localization of LC3 in astrocytes, immunofluorescence double labeling results showed 
that LC3 and GFAP positive cells were localized in the cytoplasm and nucleus, and the co-localization decreased in the SCI group, it suggested that the increase of autophagy level after PQQ induced SCI was not caused by astrocytes.

PI3K/AKT signaling pathway is regulated by extracellular cytokines and growth factors (79), involved in a variety of physiological processes such as cell survival, migration, proliferation, differentiation, and metabolism (80). After activation, PI3K phosphorylates phosphatidylinositol (PIP) and generates PIP 3, thereby activating various downstream signaling pathways (79). Phosphorylation of PI3K/Akt inhibited the activity of Bax protein in chondrocytes and increased the expression of Bcl-2, thereby inhibiting apoptosis (81). Apoptosis and autophagy have an intricate relationship. So does PI3K/ Akt signaling pathway participate in the regulation of apoptosis and autophagy after SCI by PQQ? This topic has proved that PQQ can inhibit apoptosis after SCI by maintaining the balance of Bcl-2 and Bax, and then detect the expression of phosphorylated AKT after SCI. The results show that the protein expression level of phosphorylated AKT is up-regulated after SCI, and the protein expression level of phosphorylated AKT is down-regulated after PQQ intervention, suggesting that PI3K /AKT signaling pathway may play a role in the regulation of PQQ in SCI.

In conclusion, PQQ realizes its protective effect in SCI by improving the motor function of hind limbs, reducing the loss of neurons, and participating in the process of apoptosis and autophagy. The results at the cellular level and overall level suggested that astrocytes may also be involved in inflammation, apoptosis, and autophagy after SCI.

\section{Acknowledgments}

We thank Dr Waleed Hussein (School of Chemistry and Molecular Biosciences, The University of Queensland, Brisbane Qld 4072 Australia).

Funding: This work was supported by the National Natural Scientific Foundation of China (31370803), Public Health Program of Nantong City (MS22019001), Science and Technology Program of Zhangjiagang City (ZKS1930).

\section{Footnote}

Reporting Checklist: The authors have completed the ARRIVE reporting checklist. Available at https://dx.doi. org/10.21037/atm-21-1951
Data Sharing Statement: Available at https://dx.doi. org/10.21037/atm-21-1951

Peer Review File: Available at https://dx.doi.org/10.21037/ atm-21-1951

Conflicts of Interest: All authors have completed the ICMJE uniform disclosure form (available at https://dx.doi. org/10.21037/atm-21-1951). The authors have no conflicts of interest to declare.

Ethical Statement: The authors are accountable for all aspects of the work in ensuring that questions related to the accuracy or integrity of any part of the work are appropriately investigated and resolved. All animal care, breeding, and testing procedures were approved according to the Animal Care and Use Committee of Nantong University (License number: S20190920-003) and the Animal Care Ethics Committee of Jiangsu Province, in compliance with internationally recognized and institutional guidelines for the care and use of animals. A protocol was prepared before the study without registration.

Open Access Statement: This is an Open Access article distributed in accordance with the Creative Commons Attribution-NonCommercial-NoDerivs 4.0 International License (CC BY-NC-ND 4.0), which permits the noncommercial replication and distribution of the article with the strict proviso that no changes or edits are made and the original work is properly cited (including links to both the formal publication through the relevant DOI and the license). See: https://creativecommons.org/licenses/by-nc-nd/4.0/.

\section{References}

1. Rucker R, Chowanadisai W, Nakano M. Potential physiological importance of pyrroloquinoline quinone. Altern Med Rev 2009;14:268-77.

2. Misra HS, Rajpurohit YS, Khairnar NP. Pyrroloquinolinequinone and its versatile roles in biological processes. J Biosci 2012;37:313-25.

3. Stites TE, Mitchell AE, Rucker RB. Physiological importance of quinoenzymes and the $\mathrm{O}$-quinone family of cofactors. J Nutr 2000;130:719-27.

4. Akagawa M, Minematsu K, Shibata T, et al. Identification of lactate dehydrogenase as a mammalian pyrroloquinoline quinone (PQQ)-binding protein. Sci Rep 2016;6:26723. 
5. Goodwin PM, Anthony C. The biochemistry, physiology and genetics of PQQ and PQQ-containing enzymes. Adv Microb Physiol 1998;40:1-80.

6. Kumazawa T, Seno H, Urakami T, et al. Trace levels of pyrroloquinoline quinone in human and rat samples detected by gas chromatography/mass spectrometry. Biochim Biophys Acta 1992;1156:62-6.

7. Noji N, Nakamura T, Kitahata N, et al. Simple and sensitive method for pyrroloquinoline quinone (PQQ) analysis in various foods using liquid chromatography/ electrospray-ionization tandem mass spectrometry. J Agric Food Chem 2007;55:7258-63.

8. Yakushi T, Matsushita K. Alcohol dehydrogenase of acetic acid bacteria: structure, mode of action, and applications in biotechnology. Appl Microbiol Biotechnol 2010;86:1257-65.

9. Gómez-Manzo S, Contreras-Zentella M, GonzálezValdez A, et al. The PQQ-alcohol dehydrogenase of Gluconacetobacter diazotrophicus. Int J Food Microbiol 2008; 125:71-8.

10. Steinberg F, Stites TE, Anderson P, et al. Pyrroloquinoline quinone improves growth and reproductive performance in mice fed chemically defined diets. Exp Biol Med (Maywood) 2003;228:160-6.

11. Killgore J, Smidt C, Duich L, et al. Nutritional importance of pyrroloquinoline quinone. Science 1989;245:850-2.

12. Steinberg FM, Gershwin ME, Rucker RB. Dietary pyrroloquinoline quinone: growth and immune response in BALB/c mice. J Nutr 1994;124:744-53.

13. Harris CB, Chowanadisai W, Mishchuk DO, et al. Dietary pyrroloquinoline quinone (PQQ) alters indicators of inflammation and mitochondrial-related metabolism in human subjects. J Nutr Biochem 2013;24:2076-84.

14. Ma W, Zhang R, Huang Z, et al. PQQ ameliorates skeletal muscle atrophy, mitophagy and fiber type transition induced by denervation via inhibition of the inflammatory signaling pathways. Ann Transl Med 2019;7:440.

15. Akagawa $M$, Nakano $M$, Ikemoto K. Recent progress in studies on the health benefits of pyrroloquinoline quinone. Biosci Biotechnol Biochem 2016;80:13-22.

16. Xu F, Yu H, Liu J, et al. Pyrroloquinoline quinone inhibits oxygen/glucose deprivation-induced apoptosis by activating the PI3K/AKT pathway in cardiomyocytes. Mol Cell Biochem 2014;386:107-15.

17. Chowanadisai W, Bauerly KA, Tchaparian E, et al. Pyrroloquinoline quinone stimulates mitochondrial biogenesis through cAMP response element-binding protein phosphorylation and increased PGC-1alpha expression. J Biol Chem 2010;285:142-52.

18. Tchaparian E, Marshal L, Cutler G, et al. Identification of transcriptional networks responding to pyrroloquinoline quinone dietary supplementation and their influence on thioredoxin expression, and the JAK/STAT and MAPK pathways. Biochem J 2010;429:515-26.

19. Stites T, Storms D, Bauerly K, et al. Pyrroloquinoline quinone modulates mitochondrial quantity and function in mice. J Nutr 2006;136:390-6.

20. Bauerly K, Harris C, Chowanadisai W, et al. Altering pyrroloquinoline quinone nutritional status modulates mitochondrial, lipid, and energy metabolism in rats. PLoS One 2011;6:e21779.

21. Yamaguchi K, Sasano A, Urakami T, et al. Stimulation of nerve growth factor production by pyrroloquinoline quinone and its derivatives in vitro and in vivo. Biosci Biotechnol Biochem 1993;57:1231-3.

22. Murase K, Hattori A, Kohno M, et al. Stimulation of nerve growth factor synthesis/secretion in mouse astroglial cells by coenzymes. Biochem Mol Biol Int 1993;30:615-21.

23. Alizadeh A, Dyck SM, Karimi-Abdolrezaee S. Traumatic Spinal Cord Injury: An Overview of Pathophysiology, Models and Acute Injury Mechanisms. Front Neurol 2019;10:282.

24. Heron DE, Rajagopalan MS, Stone B, et al. Single-session and multisession CyberKnife radiosurgery for spine metastases-University of Pittsburgh and Georgetown University experience. J Neurosurg Spine 2012;17:11-8.

25. Oyinbo CA. Secondary injury mechanisms in traumatic spinal cord injury: a nugget of this multiply cascade. Acta Neurobiol Exp (Wars) 2011;71:281-99.

26. Dumont RJ, Okonkwo DO, Verma S, et al. Acute spinal cord injury, part I: pathophysiologic mechanisms. Clin Neuropharmacol 2001;24:254-64.

27. Sekhon LH, Fehlings MG. Epidemiology, demographics, and pathophysiology of acute spinal cord injury. Spine (Phila Pa 1976) 2001;26:S2-12.

28. von Leden RE, Yauger YJ, Khayrullina G, et al. Central Nervous System Injury and Nicotinamide Adenine Dinucleotide Phosphate Oxidase: Oxidative Stress and Therapeutic Targets. J Neurotrauma 2017;34:755-64.

29. Metz GA, Curt A, van de Meent H, et al. Validation of the weight-drop contusion model in rats: a comparative study of human spinal cord injury. J Neurotrauma 2000;17:1-17.

30. Basso DM, Beattie MS, Bresnahan JC. A sensitive and reliable locomotor rating scale for open field testing in rats. J Neurotrauma 1995;12:1-21.

31. Olby NJ, De Risio L, Muñana KR, et al. Development of 
a functional scoring system in dogs with acute spinal cord injuries. Am J Vet Res 2001;62:1624-8.

32. Song RB, Basso DM, da Costa RC, et al. Adaptation of the Basso-Beattie-Bresnahan locomotor rating scale for use in a clinical model of spinal cord injury in dogs. J Neurosci Methods 2016;268:117-24.

33. Beattie MS, Farooqui AA, Bresnahan JC. Review of current evidence for apoptosis after spinal cord injury. J Neurotrauma 2000;17:915-25.

34. Ren XD, Wan CX, Niu YL. Overexpression of lncRNA TCTN2 protects neurons from apoptosis by enhancing cell autophagy in spinal cord injury. FEBS Open Bio 2019;9:1223-31.

35. Elmore S. Apoptosis: a review of programmed cell death. Toxicol Pathol 2007;35:495-516.

36. Galluzzi L, Kepp O, Kroemer G. Mitochondria: master regulators of danger signalling. Nat Rev Mol Cell Biol 2012;13:780-8.

37. Zhang N, Yin Y, Xu SJ, et al. Inflammation \& apoptosis in spinal cord injury. Indian J Med Res 2012;135:287-96.

38. Wong WW, Puthalakath H. Bcl-2 family proteins: the sentinels of the mitochondrial apoptosis pathway. IUBMB Life 2008;60:390-7.

39. Yuan S, Akey CW. Apoptosome structure, assembly, and procaspase activation. Structure 2013;21:501-15.

40. Zhou K, Sansur CA, Xu H, et al. The Temporal Pattern, Flux, and Function of Autophagy in Spinal Cord Injury. Int J Mol Sci 2017;18:466.

41. Mizushima N. Autophagy: process and function. Genes Dev 2007;21:2861-73.

42. He M, Ding Y, Chu C, et al. Autophagy induction stabilizes microtubules and promotes axon regeneration after spinal cord injury. Proc Natl Acad Sci U S A 2016;113:11324-9.

43. Zhang HY, Wang ZG, Wu FZ, et al. Regulation of autophagy and ubiquitinated protein accumulation by bFGF promotes functional recovery and neural protection in a rat model of spinal cord injury. Mol Neurobiol 2013;48:452-64.

44. Nakatogawa H. Two ubiquitin-like conjugation systems that mediate membrane formation during autophagy. Essays Biochem 2013;55:39-50.

45. Birgisdottir $\AA$ B, Lamark T, Johansen T. The LIR motif crucial for selective autophagy. J Cell Sci 2013;126:3237-47.

46. Fazeli G, Wehman AM. Safely removing cell debris with LC3-associated phagocytosis. Biol Cell 2017;109:355-63.

47. Saftig P, Klumperman J. Lysosome biogenesis and lysosomal membrane proteins: trafficking meets function.
Nat Rev Mol Cell Biol 2009;10:623-35.

48. Fukuda M. Lysosomal membrane glycoproteins. Structure, biosynthesis, and intracellular trafficking. J Biol Chem 1991;266:21327-30.

49. Boman A, Svensson S, Boxer A, et al. Distinct Lysosomal Network Protein Profiles in Parkinsonian Syndrome Cerebrospinal Fluid. J Parkinsons Dis 2016;6:307-15.

50. Armstrong A, Mattsson N, Appelqvist H, et al. Lysosomal network proteins as potential novel CSF biomarkers for Alzheimer's disease. Neuromolecular Med 2014;16:150-60.

51. Sjödin S, Öhrfelt A, Brinkmalm G, et al. Targeting LAMP2 in human cerebrospinal fluid with a combination of immunopurification and high resolution parallel reaction monitoring mass spectrometry. Clin Proteomics 2016;13:4.

52. Wang L, Feng D, Liu Y, et al. Autophagy plays a protective role in motor neuron degeneration following spinal cord ischemia/reperfusion-induced spastic paralysis. Am J Transl Res 2017;9:4261-70.

53. Xie L, Yu S, Yang K, et al. Hydrogen Sulfide Inhibits Autophagic Neuronal Cell Death by Reducing Oxidative Stress in Spinal Cord Ischemia Reperfusion Injury. Oxid Med Cell Longev 2017;2017:8640284.

54. Verkhratsky A, Nedergaard M. Physiology of Astroglia. Physiol Rev 2018;98:239-389.

55. Colombo E, Farina C. Astrocytes: Key Regulators of Neuroinflammation. Trends Immunol 2016;37:608-20.

56. Cekanaviciute E, Buckwalter MS. Astrocytes: Integrative Regulators of Neuroinflammation in Stroke and Other Neurological Diseases. Neurotherapeutics 2016;13:685-701.

57. Dinarello CA. Overview of the IL-1 family in innate inflammation and acquired immunity. Immunol Rev 2018;281:8-27.

58. Naugler WE, Karin M. The wolf in sheep's clothing: the role of interleukin- 6 in immunity, inflammation and cancer. Trends Mol Med 2008;14:109-19.

59. Majid DS. Tumor necrosis factor- $\alpha$ and kidney function: experimental findings in mice. Adv Exp Med Biol 2011;691:471-80.

60. Rodríguez-Iturbe B, Pons H, Quiroz Y, et al. The immunological basis of hypertension. Am J Hypertens 2014;27:1327-37.

61. Liu XZ, Xu XM, Hu R, et al. Neuronal and glial apoptosis after traumatic spinal cord injury. J Neurosci 1997; 17:5395-406.

62. HAUGE JG. GLUCOSE DEHYDROGENASE OF BACTERIUM ANITRATUM: AN ENZYME WITH 


\section{A NOVEL PROSTHETIC GROUP. J Biol Chem 1964;239:3630-9.}

63. Hayta E, Elden H. Acute spinal cord injury: A review of pathophysiology and potential of non-steroidal antiinflammatory drugs for pharmacological intervention. J Chem Neuroanat 2018;87:25-31.

64. Quadri SA, Farooqui M, Ikram A, et al. Recent update on basic mechanisms of spinal cord injury. Neurosurg Rev 2020;43:425-41.

65. Hirakawa A, Shimizu K, Fukumitsu H, et al. Pyrroloquinoline quinone attenuates iNOS gene expression in the injured spinal cord. Biochem Biophys Res Commun 2009;378:308-12.

66. Mortazavi MM, Verma K, Deep A, et al. Chemical priming for spinal cord injury: a review of the literature part IIpotential therapeutics. Childs Nerv Syst 2011;27:1307-16.

67. Ray SK, Dixon CE, Banik NL. Molecular mechanisms in the pathogenesis of traumatic brain injury. Histol Histopathol 2002;17:1137-52.

68. Kaplan AE. Spinal cord influences on immune responses demonstrated following interruption of the spinal cord as a result of injury. Zh Vopr Neirokhir Im N N Burdenko 1977;(6):40-3.

69. Yang $Z$, Bao $Y$, Chen $W$, et al. Melatonin exerts neuroprotective effects by attenuating astro- and microgliosis and suppressing inflammatory response following spinal cord injury. Neuropeptides 2020;79:102002.

70. Yang C, Yu L, Kong L, et al. Pyrroloquinoline quinone (PQQ) inhibits lipopolysaccharide induced inflammation in part via downregulated NF- $\mathrm{kB}$ and $\mathrm{p} 38 / \mathrm{JNK}$ activation in microglial and attenuates microglia activation in lipopolysaccharide treatment mice. PLoS One 2014;9:e109502.

71. Iglesias J, Morales L, Barreto GE. Metabolic and

Cite this article as: Zhou Q, Jin H, Shi N, Gao S, Wang X, Zhu S, Yan M. Inhibit inflammation and apoptosis of pyrroloquinoline on spinal cord injury in rat. Ann Transl Med 2021;9(17):1360. doi: 10.21037/atm-21-1951
Inflammatory Adaptation of Reactive Astrocytes: Role of PPARs. Mol Neurobiol 2017;54:2518-38.

72. Allen NJ. Astrocyte regulation of synaptic behavior. Annu Rev Cell Dev Biol 2014;30:439-63.

73. Chung WS, Allen NJ, Eroglu C. Astrocytes Control Synapse Formation, Function, and Elimination. Cold Spring Harb Perspect Biol 2015;7:a020370.

74. Liu Y, Levine B. Autosis and autophagic cell death: the dark side of autophagy. Cell Death Differ 2015;22:367-76.

75. Yu D, Li M, Ni B, et al. Induction of neuronal mitophagy in acute spinal cord injury in rats. Neurotox Res 2013;24:512-22.

76. Liu J, Liu W, Yang H. Balancing Apoptosis and Autophagy for Parkinson's Disease Therapy: Targeting BCL-2. ACS Chem Neurosci 2019;10:792-802.

77. Zhang P, Ye Y, Qian Y, et al. The Effect of Pyrroloquinoline Quinone on Apoptosis and Autophagy in Traumatic Brain Injury. CNS Neurol Disord Drug Targets 2017;16:724-36.

78. Issa AR, Sun J, Petitgas C, et al. The lysosomal membrane protein LAMP2A promotes autophagic flux and prevents SNCA-induced Parkinson disease-like symptoms in the Drosophila brain. Autophagy 2018;14:1898-910.

79. Beck JT, Ismail A, Tolomeo C. Targeting the phosphatidylinositol 3-kinase (PI3K)/AKT/mammalian target of rapamycin (mTOR) pathway: an emerging treatment strategy for squamous cell lung carcinoma. Cancer Treat Rev 2014;40:980-9.

80. Kim EH, Suresh M. Role of PI3K/Akt signaling in memory CD8 T cell differentiation. Front Immunol 2013;4:20.

81. Lee SW, Lee HJ, Moon JB, et al. Purified extract from Clematis mandshurica prevents adenoviral-TRAIL induced apoptosis on rat articular chondrocytes. Am J Chin Med 2008;36:399-410. 